\title{
Anzer Vadisi ve Çevresinin Briyofit Florası (İkizdere, Rize)
}

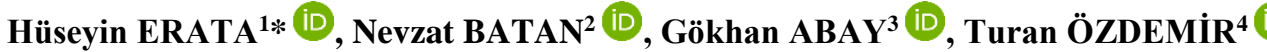 \\ ${ }^{I}$ Çanakkale Onsekiz Mart Üniversitesi, Bayramiç Meslek Yüksekokulu, Çanakkale, TÜRKIYE \\ ${ }^{2}$ Karadeniz Teknik Üniversitesi, Maçka Meslek Yüksekokulu, Trabzon, TÜRKIYE \\ ${ }^{3}$ Recep Tayyip Erdogan Üniversitesi, Mühendislik ve Mimarlık Fakültesi, Peyzaj Mimarllğı Bölümü, Rize, \\ TÜRKIYE \\ ${ }^{4}$ Karadeniz Teknik Üniversitesi, Fen Fakültesi, Biyoloji Bölümü, 61080, Trabzon, TÜRKIYE
}

\begin{abstract}
Received: 10 September $2021 \quad$ Revised: 25 September $2021 \quad$ Accepted: 29 September 2021
$\ddot{O ̈ z}$

Anzer vadisi ve çevresinin briyofit çeşitliliğinin ortaya çıkarılması amacıyla araştırma alanından Haziran ve Temmuz 2019 aylarında 33 istasyondan briyofit örnekleri toplanmıştır. Örneklerin incelenmesi sonucunda, 69 familya ve 135 cinse ait 286 karayosunu ve 52 ciğerotu taksonu olmak üzere toplamda 338 briyofit taksonu (tür, alttür ve varyete) tanımlanmıştır. Teşhis edilenler arasından Scapania obscura (Arn. \& Jens.) Schiffn., Sphagnum fimbriatum Wilson, Sphagnum papillosum Lindb., Dicranella staphylina H.Whitehouse, ve Pohlia lescuriana (Sull.) Ochi, taksonlarının Türkiye'den ikinci kez kaydı verilmiştir. Ayrıca Rize'den ilk defa kaydı verilen briyofit taksonu sayısı 88 (9' u ciğerotu, 79' u karayosunu)'dir. Yapılan bu çalışma sonucunda Rize ili için yeni olan 88 taksonun eklenmesi ile Rize ilinden tespit edilmiş olan briyofit taksonu sayıs1 424 olmuştur.
\end{abstract}

Anahtar kelimeler: Briyoflora, Ciğerotları, Karayosunları, Türkiye

\section{Bryophyte Flora of Anzer Valley and Its Surroundings (İkizdere, Rize)}

\begin{abstract}
In order to reveal the bryophyte diversity of the Anzer valley and its surroundings, bryophyte samples were collected from 33 stations in the research area between June and July 2019. As a result of the examination of the samples, a total of 338 bryophyte taxa belonging to 135 genera and 69 families were identified and from those 286 were mosses and 52 liverworts. Among the identified, Scapania obscura (Arn. \& Jens.) Schiffn., Sphagnum fimbriatum Wilson, Sphagnum papillosum Lindb., Dicranella staphylina H.Whitehouse, and Pohlia lescuriana (Sull.) Ochi, are reported for the second time from Turkey. In addition, a total of 88 bryophyte taxa (9 liverworts and 79 mosses) are new to for Rize province. As a result of this study, with the addition of 88 new taxa for Rize province, the number of bryophyte taxa determined from Rize province is 424 .
\end{abstract}

Key words: Bryoflora, Liverworts, Mosses, Turkey

\section{Giriș}

Araştırma alanı olan Anzer Vadisi ve çevresi, Rize ili İkizdere ilçesi sınırlarında yer almaktadır. Batısında Bayburt ve Trabzon, Güneyinde Erzurum, Kuzeyinde Kalkandere ve Rize merkez
Doğusunda Çayeli ve Çamlıhemşin ilçeleri yer almaktadır (Şekil 1). Araştırma alanında 3 vejetasyon tipi bulunmaktadır. 200 ile $1900 \mathrm{~m}$ aras 1 orman, 1900-2200 $\mathrm{m}$ aras subalpin, subalpin katından 3100-3200 m ye kadar olan kısımlarda ise

\footnotetext{
* Corresponding author: huseyin_erata@hotmail.com

(C) 2021 All rights reserved / Tüm haklarl saklıdır.

To cite this article: Erata H. Batan N. Abay G. Özdemir T. 2021. Anzer Vadisi ve Çevresinin Briyofit Florası (İkizdere, Rize). Anatolian Bryology. 7:2, 131-145.
}

(c) (1) () 
alpin vejetasyon vardır. Doğu Karadeniz Bölgesi'nin genelinde olduğu gibi Anzer vadisi ve çevresinde de çok sayıda derin vadiler ve bu vadilerin içerisinden akan çok sayıda akarsu mevcuttur. Bu derin vadilerde yer alan çok sayıdaki akarsu bölgenin oldukça nemli olmasına neden olmuştur. Bu iklimsel özelliklerin doğal sonucu olarak bölge ormanlarla kaplıdır (Şekil 2). İkizdere ilçesi tipik okyanus iklimine sahiptir ve hemen hemen kuraklık yaşanan mevsimi yoktur. Yıllık yağış $2254,4 \mathrm{~mm}$, yıllık ortalama sıcaklık 14,3 ${ }^{\circ} \mathrm{C}$ dir. En soğuk ay $6{ }^{\circ} \mathrm{C}$ ile Ocak, en sicak $24{ }^{\circ} \mathrm{C}$ ile Temmuz'dur (Anşin, 1981; Akman, 1999).

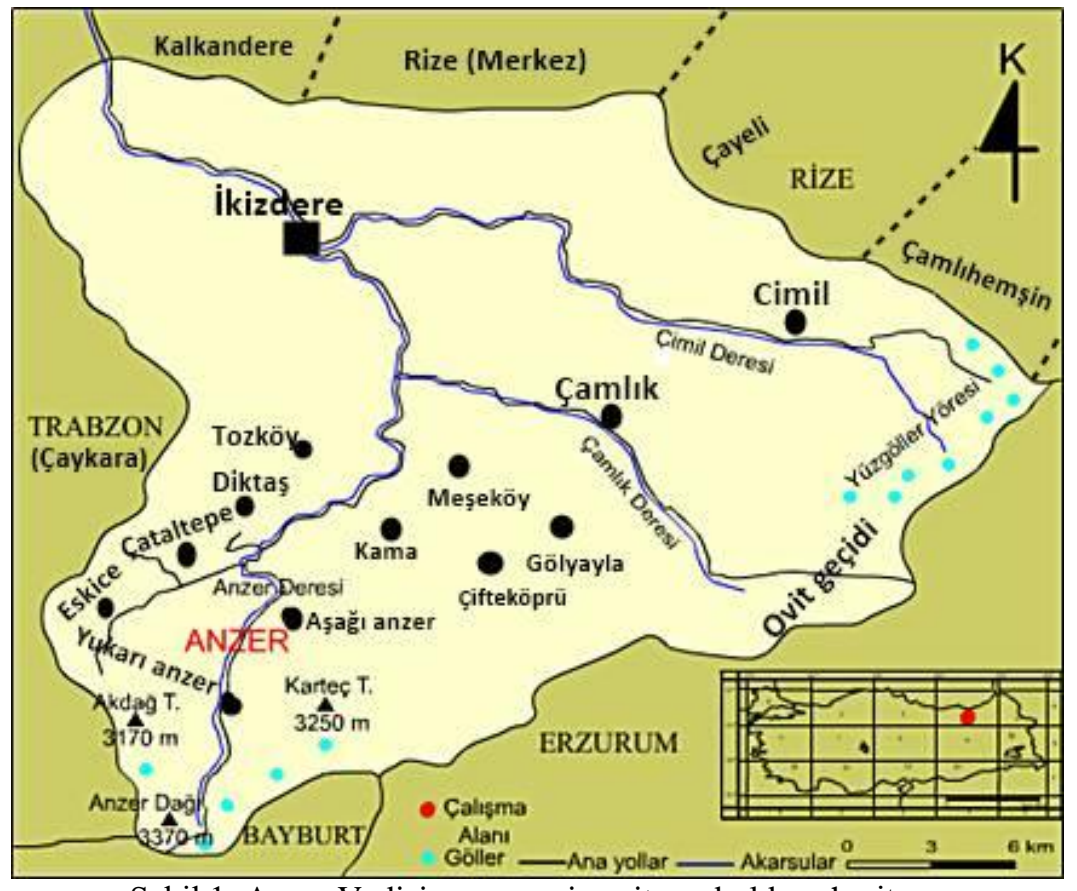

Şekil 1. Anzer Vadisi ve çevresine ait yer bulduru haritası

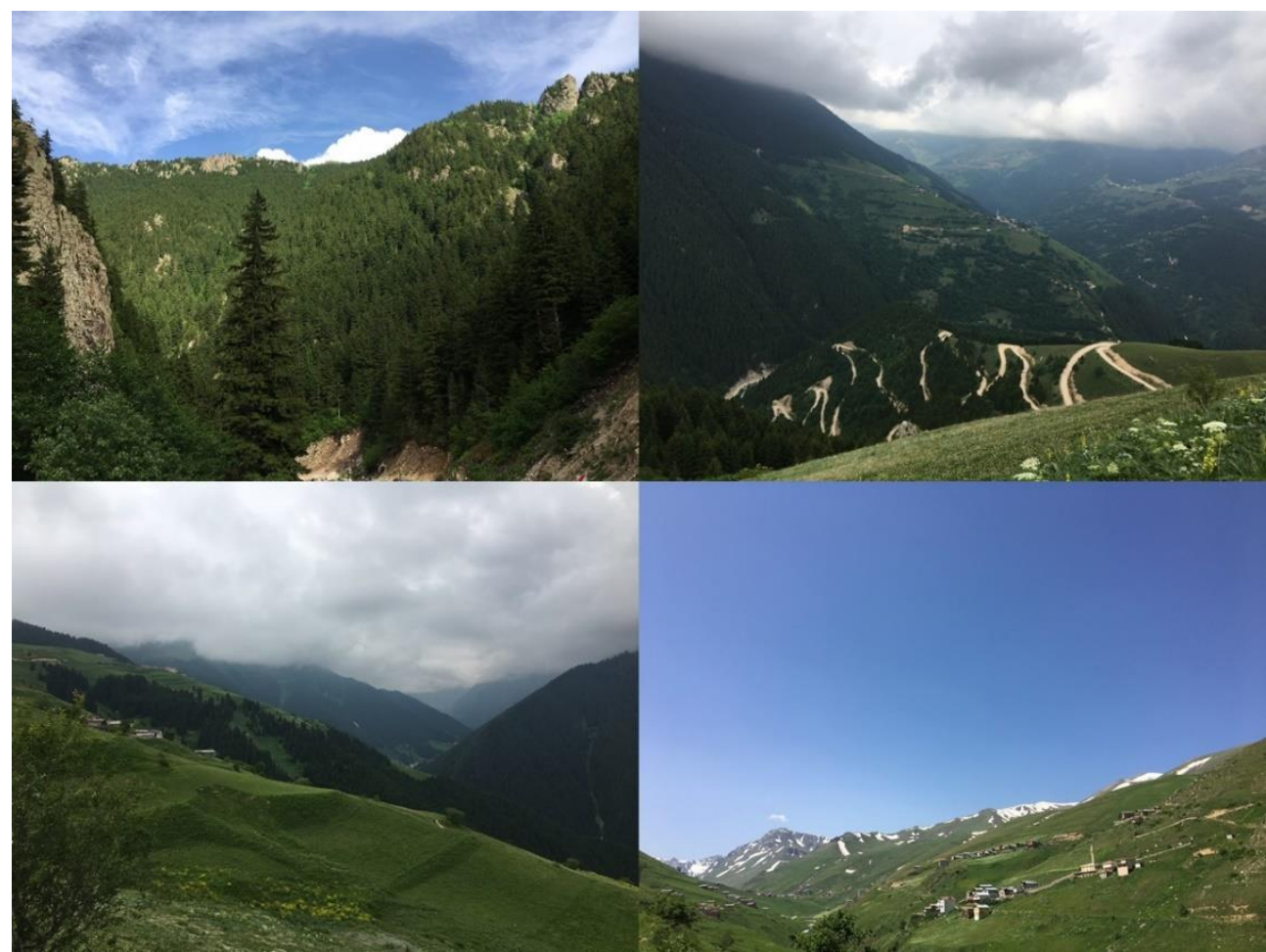

Şekil 2. Anzer Vadisi’nden bazı vejetasyon tipleri. 
Anzer vadisi İkizdere ilçesinden başlar ve 3200 metreye kadar uzanır (Şekil 1). Yaklaşık yedi ay (Ekim-Mayıs) karla kaplı olan Anzer vadisinde İkizdere ilçesine göre kışlar daha soğuk, yazlar yağışlı ve serindir. Araştırma alanının (Anzer vadisi) hakim bitki örtüsüne ait tipik bitki türleri Rhododendron ponticum L., R. luteum Sweet., Ulmus glabra Mill., Alnus glutinosa subsp. barbata (CA Mey.) Yalt., Buxus sempervirens L., Corylus avellana L., Ilex colchica Pojk., Daphne pontica L., Fagus orientalis Lipsky, Picea orientalis (L.) Link., Laurus nobilis L., Castanea sativa Mill ., Carpinus betulus L., C. orientalis Mill., Acer trautvetteri Medw., A. cappadocicum Gled., A. platanoides L., A. campestre L., Tilia rubra subsp. caucasica (Rupr.) V. Engler, Salix sp.'dir (Anşin 1981).

\section{Materyal ve Metot}

2019 yılı Haziran ve Temmuz aylarında Anzer Vadisi ve çevresinde briyofitlerin yayılış gösterebileceği değişik habitatlar ve yetişme ortamları gezilerek 33 farklı lokaliteden yaklaşık \pm 6000 briyofit örneği toplanmıştır. (Şekil 3; Tablo 1). Toplanan briyofit örnekleri 1 şı mikroskobu ve steromikroskop ile incelenmiş ve ilgili flora, revizyon ve monograflar kullanılarak teşhis edilmiştir (Nyholm, 1986, 1989, 1993, 1998; Crum ve Anderson, 1981; Paton, 1999; Smith, 1996, 2004; Hedenäs, 1992; Cortini-Pedrotti 2001, 2006; Guerra ve ark., 2006; Frey ve ark., 2006; Brugués ve ark., 2007; Casas ve ark., 2009; Brugués ve Guerra, 2015; Kürschner ve Frey, 2020). Bitki listesinin oluşturulmasında ve briyofitlere ait nomenklatürel değişiklikler ve sinonimler için Hodgetts ve ark. (2020) takip edilmiştir.

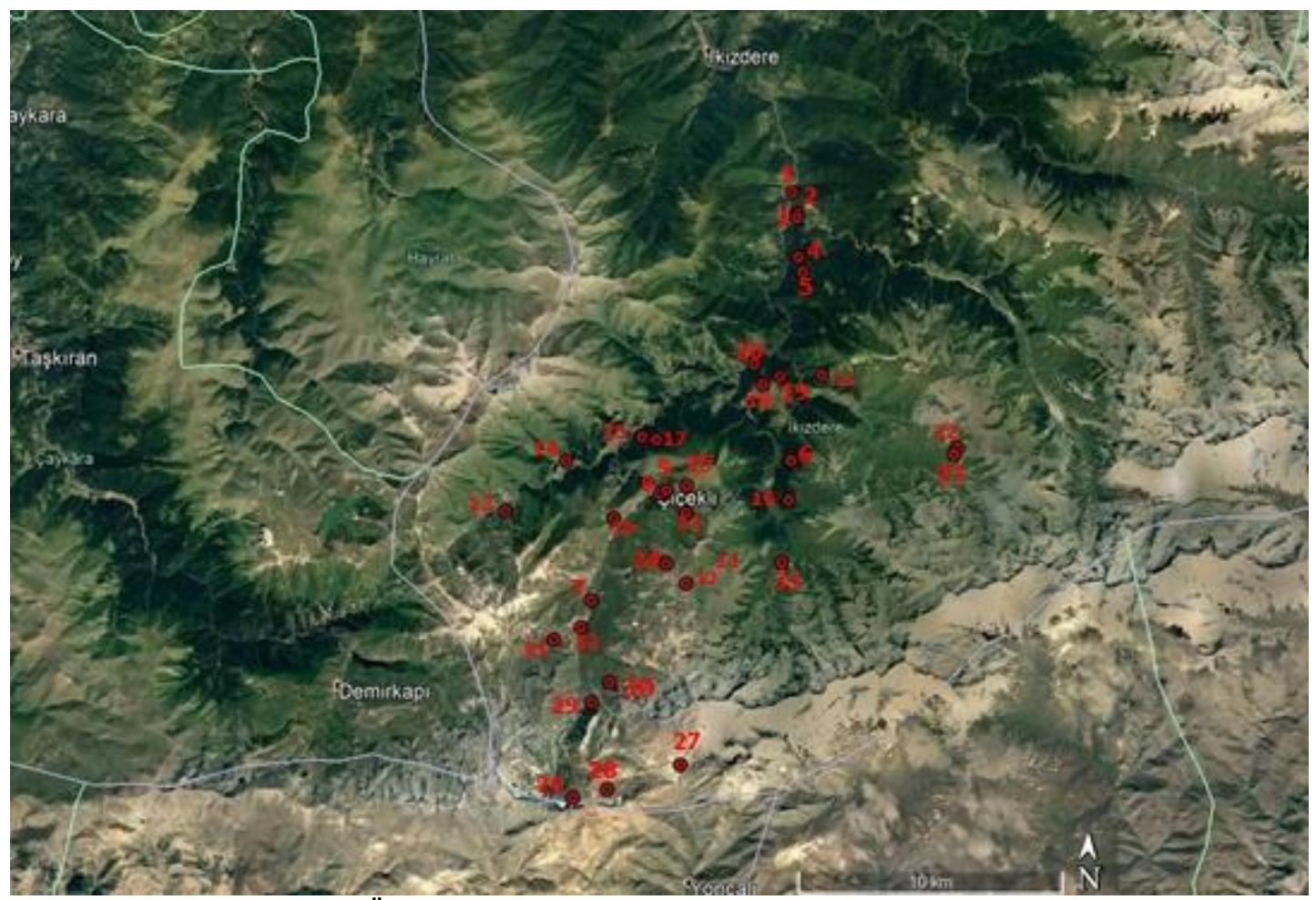

Şekil 3. Örnek toplanan lokalitelerin harita üzerindeki konumları

Tablo 1.Araştırma alanından toplanan briyofit örneklerine ait lokalite bilgileri

\begin{tabular}{|c|c|c|c|}
\hline $\begin{array}{c}\text { Sira } \\
\text { No }\end{array}$ & $\begin{array}{l}\text { Tarih/ } \\
\text { Rakım }\end{array}$ & Lokalite/ GPS Koordinatları & Vejetasyon Tipi \\
\hline 1 & $\begin{array}{l}10.06 .2019 \\
1050 \mathrm{~m}\end{array}$ & $\begin{array}{l}\text { Yerelma Köyü girişi, çeşme, } \\
\text { dere kenarı } \\
40^{\circ} 42^{\prime} 06.3^{\prime \prime} \mathrm{K}, 40^{\circ} 35^{\prime} 85.9^{\prime \prime} \mathrm{D}\end{array}$ & $\begin{array}{l}\text { Picea orientalis (L.) Link., Fagus orientalis } \\
\text { Lipsky, Corylus avellena L., Alnus glutinosa } \\
\text { (L.) Gaertner karışı orman }\end{array}$ \\
\hline 2 & $\begin{array}{l}10.06 .2019 \\
1180 \mathrm{~m}\end{array}$ & $\begin{array}{c}\text { Dereköy girişi } \\
40^{\circ} 43^{\prime} 42.8^{\prime \prime} \mathrm{K}, 40^{\circ} 35^{\prime} 65.0^{\prime \prime} \mathrm{D}\end{array}$ & $\begin{array}{l}\text { Picea orientalis (L.) Link, Fagus orientalis } \\
\text { Lipsky, Alnus glutinosa (L.) Gaertner, } \\
\begin{array}{l}\text { Castanea sativa Mill. Rubus sp. L. karış1k } \\
\text { orman }\end{array}\end{array}$ \\
\hline
\end{tabular}




\begin{tabular}{|c|c|c|c|}
\hline 3 & $\begin{array}{l}10.06 .2019 \\
1100 \mathrm{~m}\end{array}$ & $\begin{array}{l}\text { Dereköy girişi, şelale mevkii } \\
40^{\circ} 43^{\prime} 12.5^{\prime \prime} \mathrm{K}, 40^{\circ} 35^{\prime} 73.9^{\prime \prime} \mathrm{D}\end{array}$ & $\begin{array}{l}\text { Fagus orientalis Lipsky., Alnus glutinosa (L.) } \\
\text { Gaertner, Castanea sativa Mill. Corylus } \\
\text { avellena L., karışı orman }\end{array}$ \\
\hline 4 & $\begin{array}{l}10.06 .2019 \\
1181 \mathrm{~m}\end{array}$ & $\begin{array}{l}\text { Yerelma Köyü altı, orman içi } \\
40^{\circ} 42^{\prime} 24.6^{\prime \prime} \mathrm{K}, 40^{\circ} 35^{\prime} 77.8^{\prime \prime} \mathrm{D}\end{array}$ & $\begin{array}{l}\text { Picea orientalis (L.) Link., Fagus orientalis } \\
\text { Lipsky, Rhododendron ponticum L., R. luetum } \\
\text { Sweet, Carpinus betulus L., Quercus robur L. } \\
\text { karış1k orman }\end{array}$ \\
\hline 5 & $\begin{array}{l}10.06 .2019 \\
1030 \mathrm{~m}\end{array}$ & $\begin{array}{c}\text { Dereköy, dere içi } \\
40^{\circ} 42^{\prime} 77.5^{\prime \prime} \mathrm{K}, 40^{\circ} 35^{\prime} 74.8^{\prime \prime} \mathrm{D}\end{array}$ & $\begin{array}{l}\text { Picea orientalis (L.) Link., Fagus orientalis } \\
\text { Lipsky, Carpinus betulus L., Castanea sativa } \\
\text { Mill., karış1k orman }\end{array}$ \\
\hline 6 & $\begin{array}{l}11.06 .2019 \\
1714 \mathrm{~m}\end{array}$ & $\begin{array}{l}\text { Çiçekli köy yolu } \\
\text { 40³7'97.5"K, 40³1'97.6"D }\end{array}$ & $\begin{array}{l}\text { Berberis vulgaris L., Corylus avellena L., } \\
\text { Juniperus communis L., Populus tremula L. } \\
\text { karış1k orman }\end{array}$ \\
\hline 7 & $\begin{array}{c}11.06 .2019 \\
2100-2165 \\
\mathrm{~m}\end{array}$ & $\begin{array}{l}\text { Yukarı Anzer Ballıköy } \\
40^{\circ} 37^{\prime} 00.3^{\prime \prime K}, 40^{\circ} 30^{\prime} 92.7^{\prime \prime D}\end{array}$ & Kayalık alan, dere kenarı \\
\hline 8 & $\begin{array}{l}11.06 .2019 \\
1925 \mathrm{~m}\end{array}$ & $\begin{array}{l}\text { Çiçekli Köyü Yatak yaylası } \\
\text { yol ayrımı } \\
40^{\circ} 37^{\prime} 32.6^{\prime \prime K}, 40^{\circ} 32^{\prime} 45.2^{\prime \prime D}\end{array}$ & Picea orientalis (L.) Link., saf orman \\
\hline 9 & $\begin{array}{c}11.06 .2019 \\
1980 \mathrm{~m}\end{array}$ & $\begin{array}{c}\text { Yatak yaylasi yolu } \\
40^{\circ} 37^{\prime} 36.4^{\prime \prime} \mathrm{K}, 40^{\circ} 32^{\prime} 56.0^{\prime \prime} \mathrm{D}\end{array}$ & Picea orientalis (L.) Link., saf orman \\
\hline 10 & $\begin{array}{l}11.06 .2019 \\
2145 \mathrm{~m}\end{array}$ & $\begin{array}{l}\text { Yukarı Anzer Ballıköy-2 } \\
40^{\circ} 35^{\prime} 19.6^{\prime \prime K}, 40^{\circ} 31^{\prime} 00.3^{\prime \prime D}\end{array}$ & Dere kenarı \\
\hline 11 & $\begin{array}{l}12.06 .2019 \\
2600 \mathrm{~m}\end{array}$ & $\begin{array}{l}\text { Aşağı Anzer köyü, yatak } \\
\text { yaylası } \\
\text { 40³6'07.0"K, } 40^{\circ} 32^{\prime} 58.9^{\prime \prime} \mathrm{D}\end{array}$ & Alpin çayır \\
\hline 12 & $\begin{array}{l}12.06 .2019 \\
2140 \mathrm{~m}\end{array}$ & $\begin{array}{l}\text { Eskice Köyü, yukarı Haya } \\
\text { üstü } \\
40^{\circ} 37^{\prime} 05.8^{\prime \prime K}, 40^{\circ} 27^{\prime} 98.8^{\prime \prime} \mathrm{D}\end{array}$ & $\begin{array}{l}\text { Picea orientalis (L.) Link., Alnus glutinosa (L.) } \\
\text { Gaertner, karışık orman }\end{array}$ \\
\hline 13 & $\begin{array}{l}12.06 .2019 \\
2400 \mathrm{~m}\end{array}$ & $\begin{array}{l}\text { Aşağı Anzer köyü, yatak } \\
\text { yaylası-2 } \\
\text { 40³6'69.7"K, } 40^{\circ} 32^{\prime} 88.8^{\prime \prime D}\end{array}$ & $\begin{array}{l}\text { Rhododendron caucasicum Pall., Sorbus } \\
\text { aucuparia L. }\end{array}$ \\
\hline 14 & $\begin{array}{l}12.06 .2019 \\
1795-1900 \\
\mathrm{~m}\end{array}$ & $\begin{array}{c}\text { Çataltepe Köyü } \\
40^{\circ} 37^{\prime} 68.7^{\prime \prime K}, 40^{\circ} 29^{\prime} 74.7^{\prime \prime D}\end{array}$ & $\begin{array}{l}\text { Picea orientalis (L.) Link, Rhododendron } \\
\text { luteum Sweet }\end{array}$ \\
\hline 15 & $\begin{array}{c}12.06 .2019 \\
1563 \mathrm{~m}\end{array}$ & $\begin{array}{l}\text { Diktaş tarihi köprüsü } \\
40^{\circ} 38^{\prime} 40.4^{\prime \prime K}, 40^{\circ} 31^{\prime} 74.6^{\prime \prime} \mathrm{D}\end{array}$ & $\begin{array}{l}\text { Picea orientalis (L.) Link., Populus tremula L., } \\
\text { Corylus avellena L., Salix alba L. karışık } \\
\text { orman }\end{array}$ \\
\hline 16 & $\begin{array}{c}13.06 .2019 \\
1868 \mathrm{~m}\end{array}$ & $\begin{array}{l}\text { Çifteköprü Köyü üstü, } \\
\text { Ormanlık alan } \\
\text { 40³6'88.8"K, 40³6'12.1"D }\end{array}$ & Picea orientalis (L.) Link., saf orman \\
\hline 17 & $\begin{array}{c}13.06 .2019 \\
1317 \mathrm{~m}\end{array}$ & $\begin{array}{l}\text { Kama Köyü dibi } \\
40^{\circ} 39^{\prime} 73.2^{\prime \prime K}, 40^{\circ} 34^{\prime} 71.5^{\prime \prime} \mathrm{D}\end{array}$ & $\begin{array}{l}\text { Picea orientalis (L.) Link., Alnus glutinosa (L.) } \\
\text { Gaertner, karışık orman }\end{array}$ \\
\hline 18 & $\begin{array}{l}13.06 .2019 \\
1757 \mathrm{~m}\end{array}$ & $\begin{array}{l}\text { Çifteköprü köyü-Meşeköy } \\
\text { arası vadi içi } \\
\text { 40³8'16.4"K, 40³5'74.0"D }\end{array}$ & $\begin{array}{l}\text { Picea orientalis (L.) Link., Alnus glutinosa (L.) } \\
\text { Gaertner, karışı orman }\end{array}$ \\
\hline 19 & $\begin{array}{c}13.06 .2019 \\
1842 \mathrm{~m}\end{array}$ & $\begin{array}{c}\text { Meşeköy } \\
40^{\circ} 39^{\prime} 58.6^{\prime \prime K}, 40^{\circ} 35^{\prime} 53.0^{\prime \prime} \mathrm{D}\end{array}$ & Picea orientalis (L.) Link., saf orman \\
\hline 20 & $\begin{array}{l}13.06 .2019 \\
1564 \mathrm{~m}\end{array}$ & $\begin{array}{c}\text { Meşeköy altı } \\
40^{\circ} 39^{\prime} 48.0^{\prime \prime} \mathrm{K}, 40^{\circ} 34^{\prime} 86.6^{\prime \prime} \mathrm{D}\end{array}$ & $\begin{array}{l}\text { Picea orientalis (L.) Link., Corylus avellena L., } \\
\text { Carpinus betulus L., Quercus robur L., karışık } \\
\text { orman }\end{array}$ \\
\hline 21 & $\begin{array}{l}14.06 .2019 \\
1550 \mathrm{~m}\end{array}$ & $\begin{array}{c}\text { Gölyayla Köyü altı } \\
40^{\circ} 39^{\prime} 61.9^{\prime \prime} \mathrm{K}, 40^{\circ} 37^{\prime} 00.5 " \mathrm{D}\end{array}$ & $\begin{array}{l}\text { Picea orientalis (L.) Link., Salix alba L., } \\
\text { Corylus avellena L. karış1k orman }\end{array}$ \\
\hline 22 & $\begin{array}{l}14.06 .2019 \\
2450 \mathrm{~m}\end{array}$ & $\begin{array}{l}\text { Gölyayla(Kabahor) Yaylası } \\
40^{\circ} 37^{\prime} 89.8^{\prime \prime K}, 40^{\circ} 39^{\prime} 96.8^{\prime \prime D}\end{array}$ & Alpin çayır \\
\hline 23 & $\begin{array}{l}14.06 .2019 \\
2300 \mathrm{~m}\end{array}$ & $\begin{array}{l}\text { Gölyayla(Kabahor) Yaylas1-2 } \\
40^{\circ} 37^{\prime} 97.4^{\prime \prime} \mathrm{K}, 40^{\circ} 40^{\prime} 41.1^{\prime \prime D}\end{array}$ & Alpin çayır \\
\hline
\end{tabular}




\begin{tabular}{|c|c|c|c|}
\hline 24 & $\begin{array}{c}16.07 .2019 \\
2718 \mathrm{~m}\end{array}$ & $\begin{array}{c}\text { Yatak Yaylas1-1 } \\
40^{\circ} 35^{\prime} 43.8^{\prime \prime} \mathrm{K}, 40^{\circ} 32^{\prime} 91.7^{\prime \prime D}\end{array}$ & Alpin çayır \\
\hline 25 & $\begin{array}{c}16.07 .2019 \\
1985 \mathrm{~m}\end{array}$ & $\begin{array}{c}\text { Çiçekli Köyü üstü } \\
40^{\circ} 37^{\prime} 42.0^{\prime \prime K}, 40^{\circ} 32^{\prime} 88.6^{\prime \prime D}\end{array}$ & Dere içi, Picea orientalis (L.) Link., saf orman \\
\hline 26 & $\begin{array}{c}17.07 .2019 \\
2640-2590 \\
\mathrm{~m}\end{array}$ & $\begin{array}{c}\text { Arcahal } \\
40^{\circ} 30^{\prime} 90.9^{\prime \prime K}, 40^{\circ} 31^{\prime} 34.3^{\prime \prime} \mathrm{D}\end{array}$ & Alpin çayır, Rhododendron caucasicum Pall., \\
\hline 27 & $\begin{array}{l}17.07 .2019 \\
3070 \mathrm{~m}\end{array}$ & $\begin{array}{c}\text { Anzer Dağ } 1 \\
40^{\circ} 32^{\prime} 02.8^{\prime \prime} \mathrm{K}, 40^{\circ} 302^{\prime} 90.0^{\prime \prime} \mathrm{D}\end{array}$ & Kayalık alan \\
\hline 28 & $\begin{array}{c}17.07 .2019 \\
2980-2960 \\
\mathrm{~m}\end{array}$ & $\begin{array}{c}\text { Dipsiz Göl } \\
40^{\circ} 31^{\prime} 18^{\prime \prime} \mathrm{K}, 40^{\circ} 30^{\prime} 39^{\prime \prime} \mathrm{D}\end{array}$ & Göl ve kayalık alan \\
\hline 29 & $\begin{array}{c}18.07 .2019 \\
2387-2500 \\
\mathrm{~m}\end{array}$ & $\begin{array}{c}\text { Koşmer yaylası } \\
40^{\circ} 33^{\prime} 40.8^{\prime \prime} \mathrm{K}, 40^{\circ} 30^{\prime} 93.8^{\prime \prime} \mathrm{D}\end{array}$ & Alpin çayır, dere içi \\
\hline 30 & $\begin{array}{c}18.07 .2019 \\
2393-2300 \\
\mathrm{~m}\end{array}$ & $\begin{array}{c}\text { Laphazalı yaylası } \\
40^{\circ} 33^{\prime} 15.5^{\prime \prime} \mathrm{K}, 40^{\circ} 30^{\prime} 65.3^{\prime \prime} \mathrm{D}\end{array}$ & Alpin çayır, dere içi \\
\hline 31 & $\begin{array}{c}18.07 .2019 \\
2400-2430 \\
\mathrm{~m}\end{array}$ & $\begin{array}{l}\text { Kürdün Yurdu yaylası } \\
40^{\circ} 34^{\prime} 30.8^{\prime \prime} \mathrm{K}, 40^{\circ} 30^{\prime} 02.0^{\prime \prime} \mathrm{D}\end{array}$ & Alpin çayır, dere içi \\
\hline 32 & $\begin{array}{l}18.07 .2019 \\
2220 \mathrm{~m}\end{array}$ & $\begin{array}{c}\text { Ballıköy yaylası üstü } \\
40^{\circ} 34^{\prime} 46.0^{\prime \prime} \mathrm{K}, 40^{\circ} 30^{\prime} 45^{\prime \prime} \mathrm{D}\end{array}$ & Dere içi \\
\hline 33 & $\begin{array}{l}19.07 .2019 \\
2072 \mathrm{~m}\end{array}$ & $\begin{array}{c}\text { Garzavan Yaylası } \\
40^{\circ} 36^{\prime} 12.5^{\prime \prime} \mathrm{K}, 40^{\circ} 36^{\prime} 04.9^{\prime \prime} \mathrm{D}\end{array}$ & $\begin{array}{l}\text { Kayalık alan, dere içi, Picea orientalis (L.) } \\
\text { Link., saf orman }\end{array}$ \\
\hline
\end{tabular}

Floristik listede taksonların ait oldukları familyalar, taksonlara ait geçerli Latince isimleri, otörler ile birlikte alfabetik sıraya göre verilmiştir. Ayrıca, her bir takson için Henderson (1961) kareleme sistemine göre hangi karede yer aldığ 1 , toplandığ 1 bölge adı, habitat, rakım, koordinat, toplama tarihi verilmiştir. Rize ili için briyofit taksonlarının durumu ilgili literatür taranarak değerlendirilmiştir (Abay ve ark., 2016; Abay, 2017, 2018; Batan ve ark., 2018).

Teşhisi yapılan briyofitler, zarflar içerisinde muhafaza edilmek suretiyle herbaryum materyali haline getirilmiştir. Bu materyaller; Recep Tayyip Erdoğan Üniversitesi, Mühendislik ve Mimarlık Fakültesi, Peyzaj Mimarlığı Bölümünde Gökhan ABAY'ın kişisel koleksiyonunda saklanmaktadır.

\section{Bulgular}

Çalışma sonucunda 21 familya ve 30 cinse ait 52 ciğerotu taksonu ve 48 familya ve 105 cinse ait 286 karayosunu taksonu olmak üzere toplamda 69 familya ve 135 cinse ait 338 briyofit taksonu tespit edilmiştir.

\subsection{Bitki listesi}

Her takson için toplanma lokaliteleri ve substratlarının verildiği floristik listede; Türkiye'den ikinci kez kaydedilen taksonlar (*) ile, Rize ili için yeni olan taksonlar ise (\#) ile gösterilmiştir.

\section{Marchantiophyta}

Anastrophyllaceae L.Söderstr., De Roo \& Hedd. Barbilophozia barbata (Schmidel ex Schreb.) Loeske; - Lok.: A4 Rize: 8, 16, 33; Kaya üzeri.

B. hatcheri (A.Evans) Loeske, - Lok.: A4 Rize: 8, 9,33 ; toprak üzeri, çürümüş ağaç kütüğü üzeri \#B. lycopodioides (Wallr.) Loeske. - Lok.: A4 Rize: 24; toprak üzeri.

\#Gymnocolea inflata (Huds.) Dumort. - Lok.: A4

Rize: 26; 1slak toprak üzeri.

Calypogeiaceae Arnell,

Calypogeia fissa (L.) Raddi. - Lok.: A4 Rize: 26; sslak toprak üzeri.

Cephaloziaceae Mig.

Cephalozia bicuspidata (L.) Dumort. - Lok.: A4 Rize: 24, 26; toprak üzeri, sslak toprak üzeri.

Fuscocephaloziopsis catenulata (Huebener) Ván̆a \& L.Söderstr. - Lok.: A4 Rize: 29; toprak üzeri.

\#F. lunulifolia (Dumort.) Ván̆a \& L.Söderstr. Lok.: A4 Rize: 13; toprak üzeri.

Cephaloziellaceae Douin

Cephaloziella divaricata (Sm.) Schiffn. - Lok.: A4 Rize: 24, 26, 33; toprak üzeri, 1slak toprak üzeri.

Conocephalaceae Müll.Frib. ex Grolle

Conocephalum conicum (L.) Dumort. - Lok.: A4 Rize: 1, 8, 11, 21, 30; 1slak toprak üzeri, ıslak kaya üzeri.

Frullaniaceae Lorch

Frullania dilatata (L.) Dumort. - Lok.: A4 Rize: 1, 15; ağaç üzeri, kaya üzeri. 
F. tamarisci (L.) Dumort. - Lok.: A4 Rize: 1, 5, 17, 25 ; kaya üzeri.

Gymnomitriaceae H.Klinggr.

Marsupella emarginata (Ehrh.) Dumort. - Lok.: A4 Rize: 9; toprak üzeri.

Jungermanniaceae Rchb.

Jungermannia atrovirens Dumort. - Lok.: A4 Rize: 11, 26, 28, 29, 30; 1slak toprak üzeri

Mesoptychia bantriensis (Hook.) L.Söderstr. \& Váňa. - Lok.: A4 Rize: 3; toprak üzeri.

Lejeuneaceae Cavers

Lejeunea cavifolia (Ehrh.) Lindb. - Lok.: A4 Rize:

3; 1slak toprak üzeri.

Lepidoziaceae Limpr.

Telaranea europaea J.J.Engel \& G.L.Merr. - Lok.:

A4 Rize: 25; 1slak toprak üzeri

Lophocoleaceae Vanden Berghen

Chiloscyphus pallescens (Ehrh.) Dumort. - Lok.:

A4 Rize: 11, 16; toprak üzeri

C. polyanthos (L.) Corda. - Lok.: A4 Rize: 13, 24,

26, 28, 30; toprak üzeri, 1slak toprak üzeri.

Lophocolea bidentata (L.) Dumort. - Lok.: A4 Rize: 24; 1slak toprak üzeri.

L. heterophylla (Schrad.) Dumort. - Lok.: A4 Rize:

3, 13, 24; toprak üzeri, sslak toprak üzeri.

L. minor Nees. - Lok.: A4 Rize: 8, 9, 10, 11, 13, 24; toprak üzeri, sslak toprak üzeri.

Lophoziaceae Cavers

Lophozia ventricosa (Dicks.) Dumort. - Lok.: A4

Rize: 13, 16, 24, 26; toprak üzeri, 1slak toprak üzeri. \#Lophoziopsis excisa (Dicks.) Konstant. \& Vilnet. - Lok.: A4 Rize: 25; toprak üzeri.

\#L. longidens (Lindb.) Konstant. \& Vilnet. - Lok.: A4 Rize: 11, 25; 1slak toprak üzeri.

Tritomaria exsecta (Schmidel) Schiffn. ex Loeske. - Lok.: A4 Rize: 24; toprak üzeri.

Marchantiaceae Lindl.

Marchantia polymorpha L. - Lok.: A4 Rize: 10, 11, $14,16,18,21,22,23,24 ; 28,30$; 1slak toprak üzeri Metzgeriaceae H.Klinggr.

Metzgeria conjugata Lindb. - Lok.: A4 Rize: 2, 3, 25; toprak üzeri, 1slak toprak üzeri.

M. furcata (L.) Corda. - Lok.: A4 Rize: 1, 25, 33; toprak üzeri, kaya üzeri.

M. pubescens (Schrank) Raddi. - Lok.: A4 Rize: 1, 17; toprak üzeri, kaya üzeri.

Pelliaceae H.Klinggr.

Pellia epiphylla (L.) Corda. - Lok.: A4 Rize: 3, 11, 24, 28, 30; 1slak toprak üzeri

Plagiochilaceae Müll.Frib.

Pedinophyllum interruptum (Nees) Kaal. - Lok.: A4 Rize: 3, 9, 10, 13, 15, 21, 22, 28, 29, 30, 33; kaya üzeri, 1slak toprak üzeri.

Plagiochila asplenioides (L.) Dumort. - Lok.: A4 Rize: 3, 4, 8, 9, 11, 15, 16, 17, 21, 22 27, 29, 33; toprak üzeri, kaya üzeri.

$P$. porelloides (Torr. ex Nees) Lindenb. - Lok.: A4 Rize: 1, 2, 8, 11, 15, 16, 25; toprak üzeri, kaya üzeri.

\section{Porellaceae Cavers}

\#Porella cordaeana (Huebener) Moore. - Lok.: A4 Rize: 9, 15, 19, 20; toprak üzeri, kaya üzeri.

P. platyphylla (L.) Pfeiff. - Lok.: A4 Rize: 4, 17, 20,33; ağaç üzeri, kaya üzeri

Radulaceae Müll.Frib.

Radula complanata (L.) Dumort. - Lok.: A4 Rize: 17; ağaç üzeri.

R. lindenbergiana Gottsche ex C.Hartm. - Lok.: A4 Rize: 1, 5, 17, 27, 28, 29, 30; kaya üzeri, ağaç üzeri. Scapaniaceae Mig.

Diplophyllum albicans (L.) Dumort. - Lok.: A4 Rize: 2; kaya üzeri.

D. taxifolium (Wahlenb.) Dumort. - Lok.: A4 Rize: 24; toprak üzeri.

Scapania aspera M.Bernet \& Bernet. - Lok.: A4 Rize: 2; toprak üzeri.

S. compacta (Roth) Dumort. - Lok.: A4 Rize: 22; 1slak toprak üzeri

S. irrigua (Nees) Nees. - Lok.: A4 Rize: 11, 22, 24 , 26, 28, 30, 33; toprak üzeri, sslak toprak üzeri

S. nemorea (L.) Grolle. - Lok.: A4 Rize: 17; toprak üzeri

*S. obscura (Arnell \& C.E.O.Jensen) Schiffn. Lok.: A4 Rize: 24, 26; 1slak toprak üzeri.

S. subalpina (Nees ex Lindenb.) Dumort. - Lok.: A4 Rize: 24, 26; toprak üzeri, 1slak toprak üzeri

S. undulata (L.) Dumort. - Lok.: A4 Rize: 1, 21, 27; kaya üzeri, toprak üzeri.

Schistochilopsis incisa (Schrad.) Konstant. - Lok.: A4 Rize: 13, 29; toprak üzeri,

Solenostomataceae Stotler \& Crand.-Stotl.

Solenostoma gracillimum (Sm.) R.M.Schust. Lok.: A4 Rize: 26; 1slak toprak üzeri.

S. hyalinum (Lyell) Mitt. - Lok.: A4 Rize: 2; toprak üzeri.

\#S. sphaerocarpum (Hook.) Steph. - Lok.: A4 Rize: 25; 1slak toprak üzeri.

Southbyaceae Váňa, Crand.-Stotl., Stotler \& D.G.Long

\#Southbya tophacea (Spruce) Spruce. - Lok.: A4 Rize: 26; 1slak toprak üzeri.

Amblystegiaceae G.Roth

\section{Bryophyta}

Camplyliadelphus chrysophyllus (Brid.) R.S.Chopra. - Lok.: A4 Rize: 27; 1slak toprak üzeri \#Campylium bambergeri (Schimp.) Hedenäs, Schlesak \& D.Quandt. - Lok.: A4 Rize: 21; kaya üzeri

C. protensum (Brid.) Kindb. - Lok.: A4 Rize: 1, 3, $12,16,22,24,26,28,29,30,32$; sslak toprak üzeri C. stellatum (Hedw.) Lange \& C.E.O.Jensen. Lok.: A4 Rize: 18, 24, 27; 1slak kaya üzeri, ıslak toprak üzeri

Cratoneuron filicinum (Hedw.) Spruce. - Lok.: A4 Rize: 1, 4, 11, 12, 24, 25, 28, 29, 30; islak toprak üzeri 
\#Hygroamblystegium tenax (Hedw.) Jenn. - Lok.: A4 Rize: 3; 1slak toprak üzeri.

Hygrohypnum luridum (Hedw.) Jenn. - Lok.: A4 Rize: 17 ; 1slak toprak üzeri.

Palustriella commutata (Hedw.) Ochyra. - Lok.: A4 Rize: 10, 29; 1slak toprak üzeri.

P. decipiens (De Not.) Ochyra. - Lok.: A4 Rize: 16, 22; 1slak toprak üzeri.

P. falcata (Brid.) Hedenäs. - Lok.: A4 Rize: 11, 12, $18,22,23,24,32$; 1slak toprak üzeri.

\#Platyhypnum duriusculum (De Not.) Ochyra. Lok.: A4 Rize: 24, 33; 1slak toprak üzeri.

\#P. smithii (Sw.) Ochyra. - Lok.: A4 Rize: 27, 28, 30 ; 1slak toprak üzeri

Pseudoamblystegium subtile (Hedw.) Vanderp. \& Hedenäs. - Lok.: A4 Rize: 16, 17, 20; ağaç gövdesi üzeri.

Amphidiaceae M.Stech

Amphidium mougeotii (Schimp.) Schimp. - Lok.:

A4 Rize: 25; 1slak kaya üzeri

Anomodontaceae Kindb.

Anomodon viticulosus (Hedw.) Hook. \& Taylor. Lok.: A4 Rize: 1, 5; toprak üzeri, kaya üzeri.

Antitrichiaceae Ignatov \& Ignatova

Antitrichia curtipendula (Hedw.) Brid. - Lok.: A4

Rize: 1; Ağaç gövdesi üzeri

Aongstroemiaceae De Not.

Dichodontium pellucidum (Hedw.) Schimp. - Lok.:

A4 Rize: 1, 2, 3, 4, 21; 1slak toprak üzeri

Diobelonella palustris (Dicks.) Ochyra. - Lok.: A4

Rize: 11, 22, 24, 25, 26, 28, 30; 1slak toprak üzeri

Aulacomniaceae Schimp.

Aulacomnium palustre (Hedw.) Schwägr. - Lok.:

A4 Rize: 24, 26; 1slak toprak üzeri

Bartramiaceae Schwägr

Bartramia halleriana Hedw. - Lok.: A4 Rize: 1, 2 , $9,15,16,17,20,21,28,30,33$; kaya üzeri

B. ithyphylla Brid. - Lok.: A4 Rize: 9, 10, 11, 13, 24, 29; kaya üzeri

Philonotis caespitosa Jur. - Lok.: A4 Rize: 3, 26; sslak toprak üzeri

P. calcarea (Bruch \& Schimp.) Schimp. - Lok.: A4

Rize: 12 ; 1slak toprak üzeri.

P. capillaris Lindb. - Lok.: A4 Rize: 28, 30; 1slak toprak üzeri.

P. fontana (Hedw.) Brid. - Lok.: A4 Rize: 11, 14, 16, 18, 24, 31, 32, 33; 1slak toprak üzeri.

P. seriata Mitt. - Lok.: A4 Rize: 10, 19, 22, 29, 33; sslak toprak üzeri

Brachytheciaceae Schimp.

Brachytheciastrum collinum (Schleich. ex Müll.Hal.) Ignatov \& Huttunen. - Lok.: A4 Rize: 30 ; toprak üzeri.

B. velutinum (Hedw.) Ignatov \& Huttunen. - Lok.: A4 Rize: 9, 13, 16, 19, 21, 29, 33; kaya üzeri, toprak üzeri.
Brachythecium albicans (Hedw.) Schimp. - Lok.: A4 Rize: 11, 14, 16, 21, 22, 24, 33; kaya üzeri, toprak üzeri.

\#B. campestre (Müll.Hal.) Schimp. - Lok.: A4

Rize: 11; toprak üzeri.

B. capillaceum (F.Weber \& D.Mohr) Giacom. Lok.: A4 Rize: 12; 1slak toprak üzeri.

B. glareosum (Bruch ex Spruce) Schimp. - Lok.:

A4 Rize: 5; toprak üzeri.

B. mildeanum (Schimp.) Schimp. - Lok.: A4 Rize:

10, 12, 19, 27; toprak üzeri.

B. rivulare Schimp. - Lok.: A4 Rize: 3, 7, 9, 14, 16, 18, 24, 28, 29, 30, 33; 1slak toprak üzeri

B. rutabulum (Hedw.) Schimp. - Lok.: A4 Rize: 1, $4,10,11,15,17,19,21,23,24,26,27,28,30,32$; sslak toprak üzeri.

B. salebrosum (Hoffm. ex F.Weber \& D.Mohr) Schimp. - Lok.: A4 Rize: 16; çürümüş ağaç kütüğü üzeri.

\#B. tommasinii (Sendtn. ex Boulay) Ignatov \& Huttunen. - Lok.: A4 Rize: 10; 1slak toprak üzeri

Eurhynchiastrum pulchellum (Hedw.) Ignatov \& Huttunen. - Lok.: A4 Rize: 9, 11, 17, 27; toprak üzeri.

Eurhynchium angustirete (Broth.) T.J.Kop. - Lok.:

A4 Rize: 1, 2, 8, 9, 15, 17; toprak üzeri

E. striatum (Hedw.) Schimp. - Lok.: A4 Rize: 21; kaya üzeri.

Homalothecium lutescens (Hedw.) H.Rob. - Lok.: A4 Rize: 2, 10, 15, 17, 20 25, 33; kaya üzeri.

\#H. philippeanum (Spruce) Schimp. - Lok.: A4 Rize: 5, 8, 9, 11, 14, 16, 17; kaya üzeri

H. sericeum (Hedw.) Schimp. - Lok.: A4 Rize: 1, $5,15,16,20,33$; kaya üzeri

Oxyrrhynchium hians (Hedw.) Loeske. - Lok.: A4

Rize: 21 ; 1slak toprak üzeri

Plasteurhynchium striatulum (Spruce) M.Fleisch. Lok.: A4 Rize: 1; toprak üzeri.

Rhynchostegiella tenella (Dicks.) Limpr. - Lok.: A4 Rize: 9, 20; kaya üzeri.

Rhynchostegium confertum (Dicks.) Schimp. Lok.: A4 Rize: 22; toprak üzeri.

R. riparoides (Hedw.) Cardot. - Lok.: A4 Rize: 29; 1slak toprak üzeri.

\#Sciuro-hypnum flotowianum (Sendtn.) Ignatov \& Huttunen. - Lok.: A4 Rize: 1, 5, 17; toprak üzeri. \#S. latifolium (Kindb.) Ignatov \& Huttunen. - Lok.: A4 Rize: 17; 1slak toprak üzeri

S. populeum (Hedw.) Ignatov \& Huttunen. - Lok.: A4 Rize: 1, 14, 15, 16, 17, 18, 19; kaya üzeri.

S. reflexum (Starke) Ignatov \& Huttunen. - Lok.: A4 Rize: 24; toprak üzeri.

Bryaceae Schwägr

\#Anomobryum concinnatum (Spruce) Lindb. Lok.: A4 Rize: 27; toprak üzeri.

Bryum argenteum Hedw. - Lok.: A4 Rize: 6, 12, 15, 33; toprak üzeri, kaya üzeri. 
\#B. dichotomum Hedw. - Lok.: A4 Rize: 7, 10, 24, 29; kaya üzeri, toprak üzeri.

\#Imbribryum alpinum (Huds. ex With.) N.Pedersen. - Lok.: A4 Rize: 12, 13, 15, 22, 24, 25, 27, 31; toprak üzeri.

I. mildeanum (Jur.) J.R.Spence. - Lok.: A4 Rize: 7. 12, 19, 22, 25, 27; toprak üzeri.

Ptychostomum capillare (Hedw.) Holyoak \& N.Pedersen. - Lok.: A4 Rize: 1, 20, 27; toprak üzeri \#P. compactum Hornsch. - Lok.: A4 Rize: 24, 29; kaya üzeri \#P. creberrimum (Taylor) J.R.Spence \& H.P.Ramsay. - Lok.: A4 Rize: 2, 8, 17, 21, 22, 29, 33; toprak üzeri

\#P. donianum (Grev.) Holyoak \& N.Pedersen. Lok.: A4 Rize: 11; 1slak kaya üzeri.

P. elegans (Nees) D.Bell \& Holyoak. - Lok.: A4 Rize: 13, 15, 19, 24, 33; toprak üzeri

\#P. funkii (Schwägr.) J.R.Spence. - Lok.: A4 Rize: 28, 30; toprak üzeri

P. imbricatulum (Müll.Hal.) Holyoak \& N.Pedersen. - Lok.: A4 Rize: 7, 11, 22; kaya üzeri, 1slak toprak üzeri.

P. intermedium (Brid.) J.R.Spence. - Lok.: A4 Rize: 29; toprak üzeri.

P. moravicum (Podp.) Ros \& Mazimpaka. - Lok.: A4 Rize: 1, 4, 5, 6, 7, 8, 13, 16, 17, 20, 21; toprak üzeri, kaya üzeri.

P. pallens (Sw. ex anon.) J.R.Spence. - Lok.: A4 Rize: 29, 33; toprak üzeri.

\#P. pallescens (Schleich. ex Schwägr.) J.R.Spence. - Lok.: A4 Rize: 3; toprak üzeri.

P. pseudotriquetrum (Hedw.) J.R.Spence \&H.P.Ramsay ex Holyoak \& N.Pedersen var. pseudotriquetrum. - Lok.: A4 Rize: 1, 3, 7, 11, 12, $14,16,18,22,24,27,28,29,30,31$, 33; 1slak kaya üzeri, 1slak toprak üzeri.

\#P. pseudotriquetrum var. bimum (Schreb.) Holyoak \& N.Pedersen. - Lok.: A4 Rize: 6, 7, 16, 22, 24; 1slak toprak üzeri.

\#P. rubens (Mitt.) Holyoak \& N.Pedersen. - Lok.: A4 Rize: 16; 1slak toprak üzeri

P. schleicheri (DC.) J.R.Spence ex D.Bell \& Holyoak. - Lok.: A4 Rize: 11, 14, 19, 24; rslak toprak üzeri

\#P. weigelii (Biehler) J.R.Spence. - Lok.: A4 Rize:

24; 1slak toprak üzeri.

Rhodobryum ontariense (Kindb.) Kindb. - Lok.: A4

Rize: 2, 4, 16, 23; toprak üzeri.

Calliergonaceae Vanderp., Hedenäs, C.J.Cox \& A.J.Shaw

Sarmentypnum exannulatum (Schimp.) Hedenäs. Lok.: A4 Rize: 26; su içi.

Straminergon stramineum (Dicks. ex Brid.) Hedenäs. - Lok.: A4 Rize: 24; su içi.

\#Warnstorfia fluitans (Hedw.) Loeske. - Lok.: A4

Rize: 26; su içi.

Climaciaceae Kindb.
Climacium dendroides (Hedw.) F.Weber \& D.Mohr. - Lok.: A4 Rize: 10, 11, 15, 18; rslak toprak üzeri.

Dicranaceae Schimp.

Dicranum bonjeanii De Not. - Lok.: A4 Rize: 10, 24, 26, 33; kaya üzeri, sslak toprak üzeri

\#D. brevifolium (Lindb.) Lindb. - Lok.: A4 Rize:

16 ; çürümüş ağaç üzeri.

\#D. elongatum Schleich. ex Schwägr. - Lok.: A4 Rize: 11; kaya üzeri.

D. flexicaule Brid. - Lok.: A4 Rize: 2, 8, 9, 11; Çürümüş ağaç kütügü üzeri, toprak üzeri

\#D. leioneuron Kindb. - Lok.: A4 Rize: 24; 1slak toprak üzeri.

D. polysetum Sw. ex anon. - Lok.: A4 Rize: 8, 22, 29; 1slak toprak üzeri, toprak üzeri.

D. scoparium Hedw. - Lok.: A4 Rize: 1, 2, 4, 8, 9 , $11,13,15,17,20,24,26,33$; çürümüş ağaç kütüğü üzeri, toprak üzeri

\#D. spadiceum J.E.Zetterst. - Lok.: A4 Rize: 10, 13, 24, 27, 29; toprak üzeri, sslak toprak üzeri

Dicranellaceae M.Stech

Dicranella heteromalla (Hedw.) Schimp. - Lok.: A4 Rize: 2, 15, 26; toprak üzeri

*D. staphylina H.Whitehouse. - Lok.: A4 Rize: 33; 1slak toprak üzeri

\#D. varia (Hedw.) Schimp. - Lok.: A4 Rize: 2, 13, 26; toprak üzeri, 1slak toprak üzeri.

Distichiaceae Schimp.

Distichium capillaceum (Hedw.) Bruch \& Schimp.

- Lok.: A4 Rize: 10, 13, 25, 29; toprak üzeri

Ditrichaceae Limpr.

Ceratodon purpureus (Hedw.) Brid. - Lok.: A4 Rize: 3, 5, 8, 10, 14, 15, 18, 19, 22, 24, 28, 30, 33; toprak üzeri

Ditrichum heteromallum (Hedw.) E.Britton. - Lok.: A4 Rize: 3; toprak üzeri.

Encalyptaceae Schimp.

Encalypta ciliata Hedw. - Lok.: A4 Rize: 8, 9, 10, 15,21 ; kaya çatlağı içi, kaya üzeri.

\#E. microstoma Bals.-Criv. \& De Not. - Lok.: A4

Rize: 25; kaya çatlağındaki toprak üzeri

E. streptocarpa Hedw. - Lok.: A4 Rize: 5, 6, 17, 21, 25; kaya çatlağındaki toprak üzeri, toprak üzeri, kaya üzeri.

Entodontaceae Kindb.

Entodon concinnus (De Not.) Paris. - Lok.: A4 Rize: 4, 5, 17, 20, 21, 25; toprak üzeri,

Fissidentaceae Schimp.

Fissidens adianthoides Hedw. - Lok.: A4 Rize: 1, 12; 1slak kaya üzeri, 1slak toprak üzeri.

F. bryoides Hedw. - Lok.: A4 Rize: 29; kaya çatlakları içinde.

F. dubius P.Beauv. - Lok.: A4 Rize: 28, 30; 1slak toprak üzeri.

Fontinalaceae Schimp.

Fontinalis antipyretica Hedw. - Lok.: A4 Rize: 22, 32; su içi. 
\#F. squamosa Hedw. - Lok.: A4 Rize: 32; su içi. Funariaceae Schwägr.

Funaria hygrometrica Hedw. - Lok.: A4 Rize: 18; kaya üzeri.

\section{Grimmiaceae Arn.}

Grimmia alpestris (F.Weber \& D.Mohr) Schleich.

- Lok.: A4 Rize: 10, 12, 22, 24, 25, 26, 29, 31, 33;

kaya üzeri.

\#G. anodon Bruch \& Schimp. - Lok.: A4 Rize: 13; kaya üzeri.

\#G. anomala Hampe ex Schimp. - Lok.: A4 Rize: 18, 24; kaya üzeri.

\#G. caespiticia (Brid.) Jur. - Lok.: A4 Rize: 10; kaya üzeri.

G. decipiens (Schultz) Lindb. - Lok.: A4 Rize: 22, 25; kaya üzeri.

\#G. donniana Sm. - Lok.: A4 Rize: 18, 28, 30; kaya üzeri

G. elatior Bruch ex Bals.-Criv. \& De Not. - Lok.:

A4 Rize: 1, 6, 10, 15, 17; kaya üzeri.

G. funalis (Schwägr.) Bruch \& Schimp. - Lok.: A4

Rize: 8, 10, 20, 21, 25, 29, 33; kaya üzeri.

G. hartmanii Schimp. - Lok.: A4 Rize: 1, 8, 10, 11, 16, 18, 22, 33; kaya üzeri

G. laevigata (Brid.) Brid. - Lok.: A4 Rize: 6; kaya üzeri

\#G. lisae De Not. - Lok.: A4 Rize: 10, 18; kaya üzeri.

G. longirostris Hook. - Lok.: A4 Rize: 31; kaya üzeri

G. montana Bruch \& Schimp. - Lok.: A4 Rize: 6, 11, 13, 23; kaya üzeri.

\#G. orbicularis Bruch ex Wilson. - Lok.: A4 Rize: 5, 27; kaya üzeri.

G. ovalis (Hedw.) Lindb. - Lok.: A4 Rize: 6, 10, 13, $15,17,18,20,24$; kaya üzeri.

G. pulvinata (Hedw.) Sm. - Lok.: A4 Rize: 31; kaya üzeri.

\#G. unicolor Hook. - Lok.: A4 Rize: 27; kaya üzeri. Racomitrium affine (F.Weber \& D.Mohr) Lindb. Lok.: A4 Rize: 2, 26; kaya üzeri.

$R$. aquaticum (Brid. ex Schrad.) Brid. - Lok.: A4

Rize: 2, 3; kaya üzeri

$R$. canescens (Hedw.) Brid. - Lok.: A4 Rize: 1, 3, $4,8,14,20,24,26$; kaya üzeri

$R$. elongatum Ehrh. ex Frisvoll. - Lok.: A4 Rize: 4, 5, 33; kaya üzeri.

$R$. ericoides (Brid.) Brid. - Lok.: A4 Rize: 11, 13, $15,16,18,21,22,23,24,25,26$; kaya üzeri.

R. macounii Kindb. - Lok.: A4 Rize: 1, 10, 16, 29; kaya üzeri.

\#R. sudeticum (Funck) Bruch \& Schimp. - Lok.: A4 Rize: 2, 4, 16; kaya üzeri.

\#Schistidium agassizii Sull. \& Lesq. - Lok.: A4 Rize: 27; kaya üzeri.

S. apocarpum (Hedw.) Bruch \& Schimp. - Lok.: A4 Rize: 3, 4, 5, 16, 21; kaya üzeri.
S. atrofuscum (Schimp.) Limpr. - Lok.: A4 Rize: 25, 29; kaya üzeri.

\#S. brunnescens Limpr. subsp. brunnescens. - Lok.: A4 Rize: 28, 30; kaya üzeri.

\#S. brunnescens subsp. griseum (Nees \& Hornsch.) H.H.Blom. - Lok.: A4 Rize: 25; kaya üzeri.

S. confertum (Funck) Bruch \& Schimp. - Lok.: A4 Rize: 5, 6, 10, 12, 14, 17, 18, 22, 23, 33; kaya üzeri. \#S. confusum H.H.Blom. - Lok.: A4 Rize: 5, 6, 10, $12,14,17,18,22,23,33$; kaya üzeri.

S. crassipilum H.H.Blom. - Lok.: A4 Rize: 33; kaya üzeri.

\#S. dupretii (Thér.) W.A.Weber. - Lok.: A4 Rize: 1, 13, 17, 24; kaya üzeri

\#S. elegantulum H.H.Blom. - Lok.: A4 Rize: 1; kaya üzeri.

S. flaccidum (De Not.) Ochyra. - Lok.: A4 Rize: 6; kaya üzeri.

S. papillosum Culm. - Lok.: A4 Rize: 1, 3, 4, 6, 10, 12, 14, 15, 16, 17, 18, 20, 21, 26, 33; kaya üzeri.

\#S. platyphyllum (Mitt.) H.Perssc - Lok.: A4 Rize: 22, 23; kaya üzeri.

\#S. rivulare (Brid.) Podp. - Lok.: A4 Rize: 7, 28, 29, 30; kaya üzeri

S. trichodon (Brid.) Poelt. - Lok.: A4 Rize: 18; kaya üzeri.

Habrodontaceae Schimp.

Habrodon persupillus (De Not.) Lindb. - Lok.: A4 Rize: 9; ağaç gövdesi üzeri.

Hedwigiaceae Schimp.

Hedwigia ciliata (Hedw.) P.Beauv. - Lok.: A4 Rize: 1, 6, 12, 17, 21; kaya üzeri.

H. emodica Hampe ex Müll.Hal. - Lok.: A4 Rize: $6,16,18,20,24$; kaya üzeri.

Hylocomiaceae M.Fleisch.

Hylocomiadelphus triquetrus (Hedw.) Ochyra \& Stebel. - Lok.: A4 Rize: 1, 2, 4, 9, 11, 13, 15, 16, 17, 20, 21, 25, 33; toprak üzeri.

Hylocomium splendens (Hedw.) Schimp. - Lok.: A4 Rize: 1, 2, 4, 7, 8, 9, 13, 15, 16, 17, 20, 21, 25; toprak üzeri.

Pleurozium schreberi (Willd. ex Brid.) Mitt. - Lok.: A4 Rize: 2; toprak üzeri.

\#Rhytidiadelphus subpinnatus (Lindb.) T.J.Kop. Lok.: A4 Rize: 24; 1slak toprak üzeri

Hymenolomataceae Ignatov \& Fedosov

Hymenoloma crispulum (Hedw.) Ochyra. - Lok.: A4 Rize: 7, 11, 24, 26, 29; kaya üzeri.

Hypnaceae Schimp.

Hypnum andoi A.J.E.Sm. - Lok.: A4 Rize: 5, 14, 15, 16, 17, 20, 21, 25; kaya üzeri.

H. cupressiforme Hedw. var. cupressiforme. - Lok.: A4 Rize: 1, 2, 3, 4, 7, 8, 15, 16, 17, 18, 20, 21; kaya üzeri, toprak üzeri, ağaç gövdesi üzeri.

H. cupressiforme var. filiforme Brid. - Lok.: A4 Rize: 1, 2, 8, 15, 17, 20; toprak üzeri, kaya üzeri, ağaç gövdesi üzeri 
H. cupressiforme var. lacunosum Brid. - Lok.: A4 Rize: $1,3,5,8,12,16,17,29$; kaya üzeri, çürümüş ağaç kütüğü üzeri, toprak üzeri.

H. jutlandicum Holmen \& E.Warncke. - Lok.: A4 Rize: 1, 4, 17, 18; toprak üzeri, kaya üzeri

\#H. resupinatum Taylor. - Lok.: A4 Rize: 1, 8, 9, $16,17,18,20,21$; kaya üzeri, toprak üzeri, ağaç kütüğü üzeri

Lembophyllaceae Broth.

Isothecium alopecuroides (Lam. ex Dubois) Isov. Lok.: A4 Rize: 1, 2, 4, 6, 7, 8, 9, 15, 16, 17, 20, 21, 22 ; toprak üzeri, kaya üzeri.

Leucobryaceae Schimp.

Campylopus subulatus Schimp. ex Milde. - Lok.:

A4 Rize: 16; kaya üzeri.

Leucodontaceae Schimp.

Leucodon sciuroides (Hedw.) Schwägr. - Lok.: A4

Rize: 1, 2, 4, 6, 7, 14, 15, 16, 17, 20; ağaç gövdesi üzeri, kaya üzeri.

Mniaceae Schwägr.

Mnium hornum Hedw. - Lok.: A4 Rize: 1; 1slak toprak üzeri.

M. marginatum (Dicks.) P.Beauv. - Lok.: A4 Rize: 7; 1slak toprak üzeri.

\#M. spinosum (Voit) Schwägr. - Lok.: A4 Rize: 1, $8,9,13,16,17,20,24,29$; 1slak toprak üzeri, toprak üzeri.

\#M. spinulosum Bruch \& Schimp. - Lok.: A4 Rize: 27; 1slak toprak üzeri.

M. stellare Hedw. - Lok.: A4 Rize: 1, 17; toprak üzeri, kaya üzeri.

\#M. thomsonii Schimp. - Lok.: A4 Rize: 21; 1slak toprak üzeri.

Plagiomnium affine (Blandow ex Funck) T.J.Kop. - Lok.: A4 Rize: 2, 4, 9, 11, 16, 17, 20, 21; toprak üzeri, 1slak toprak üzeri.

P. cuspidatum (Hedw.) T.J.Kop. - Lok.: A4 Rize: $4,5,14,15,18,19,29$; 1slak toprak üzeri, toprak üzeri

P. elatum (Bruch \& Schimp.) T.J.Kop. - Lok.: A4

Rize: 4, 9, 19; toprak üzeri.

P. ellipticum (Brid.) T.J.Kop. - Lok.: A4 Rize: 4, 9, 19; toprak üzeri.

P. medium (Bruch \& Schimp.) T.J.Kop. - Lok.: A4

Rize: 4, 9, 19; toprak üzeri.

P. rostratum (Schrad.) T.J.Kop. - Lok.: A4 Rize:

32; 1slak toprak üzeri.

P. undulatum (Hedw.) T.J.Kop. - Lok.: A4 Rize: 1, 3, 4, 16, 17, 18; 1slak toprak üzeri

Pohlia cruda (Hedw.) Lindb. - Lok.: A4 Rize: 13, 24,25 ; 1slak toprak üzeri.

*P. lescuriana (Sull.) Ochi. - Lok.: A4 Rize: 3; 1slak toprak üzeri.

P. ludwigii (Spreng. ex Schwägr.) Broth. - Lok.: A4

Rize: 27; kaya çatlakları içindeki toprak üzeri

P. nutans (Hedw.) Lindb. - Lok.: A4 Rize: 11; toprak üzeri.
P. wahlenbergii (F.Weber \& D.Mohr) A.L.Andrews. - Lok.: A4 Rize: 7, 29; toprak üzeri \#Rhizomnium magnifolium (Horik.) T.J.Kop. Lok.: A4 Rize: 7, 8, 22, 29, 33; 1slak toprak üzeri. \#R. pseudopunctatum (Bruch \& Schimp.) T.J.Kop. - Lok.: A4 Rize: 8, 18; 1slak toprak üzeri,

R. punctatum (Hedw.) T.J.Kop. - Lok.: A4 Rize: 1, $3,7,8,16,18,22,23,24,27,29$; 1slak toprak üzeri Myuriaceae M.Fleisch.

Ctenidium molluscum (Hedw.) Mitt. - Lok.: A4 Rize: 2, 3, 22; kaya üzeri, toprak üzeri

Neckeraceae Schimp.

Alleniella besseri (Lobarz.) S.Olsson, Enroth \& D.Quandt. - Lok.: A4 Rize: 20; kaya üzeri

A. complanata (Hedw.) S.Olsson, Enroth \& D.Quandt. - Lok.: A4 Rize: 1, 2, 5, 16, 17, 20; kaya üzeri, ağaç gövdesi üzeri

Exsertotheca crispa (Hedw.) S.Olsson, Enroth \& D.Quandt. - Lok.: A4 Rize: 1, 5; ağaç gövdesi üzeri Pseudanomodon attenuatus (Limpr.) Ignatov \& Fedosov. - Lok.: A4 Rize: 1, 4, 5, 16, 17, 20; kaya üzeri

Thamnobryum alopecurum (Hedw.) Gangulee. Lok.: A4 Rize: 1; 1slak toprak üzeri.

Orthotrichaceae Arn.

Lewinskya affinis (Schrad. ex Brid.) F.Lara, Garilleti \& Goffinet. - Lok.: A4 Rize: 6; kaya üzeri. L. rupestris (Schleich. ex Schwägr.) F.Lara, Garilleti \& Goffinet. - Lok.: A4 Rize: 1, 5, 6, 7, 12, 14, 17, 18, 20, 21, 24; ağaç üzeri, 1slak toprak üzeri, kaya üzeri.

L. speciosa (Nees) F.Lara, Garilleti \& Goffinet. Lok.: A4 Rize: 1, 2, 4, 9, 15, 20; kaya üzeri, toprak üzeri, ağaç üzeri.

L. striata (Hedw.) F.Lara, Garilleti \& Goffinet. Lok.: A4 Rize: 6, 9, 17; kaya üzeri

Orthotrichum alpestre Bruch \& Schimp. - Lok.: A4 Rize: 15; kaya üzeri.

O. anomalum Hedw. - Lok.: A4 Rize: 21; kaya üzeri

O. pallens Bruch ex Brid. - Lok.: A4 Rize: 6, 17, 20, 21; kaya üzeri.

\#O. pulchellum Brunt. - Lok.: A4 Rize: 17, 25; kaya üzeri.

\#O. tenellum Bruch ex Brid. - Lok.: A4 Rize: 6; kaya üzeri

Ulota crispa (Hedw.) Brid. - Lok.: A4 Rize: 21; ağaç gövdesi üzeri.

\#U. crispula Bruch. - Lok.: A4 Rize: 1, 3, 4, 20, 21;

kaya üzeri, ağaç gövdesi üzeri

Plagiotheciaceae M.Fleisch.

Plagiothecium cavifolium (Brid.) Z.Iwats. - Lok.:

A4 Rize: 1, 11; 1slak toprak üzeri

P. denticulatum (Hedw.) Schimp. - Lok.: A4 Rize: 22, 29, 33; 1slak toprak üzeri

P. laetum Schimp. - Lok.: A4 Rize: 13, 16; toprak üzeri, kaya üzeri 
P. succulentum (Wilson) Lindb. - Lok.: A4 Rize: 7, 13, 15, 21, 24, 29; toprak üzeri, kaya üzeri \#Pseudotaxiphyllum elegans (Brid.) Z.Iwats. Lok.: A4 Rize: 1; 1slak toprak üzeri.

Polytrichaceae Schwägr.

Atrichum tenellum (Röhl.) Bruch \& Schimp. - Lok.:

A4 Rize: 22; toprak üzeri

A. undulatum (Hedw.) P.Beauv. - Lok.: A4 Rize: 2,

7, 18; toprak üzeri.

Pogonatum aloides (Hedw.) P.Beauv. - Lok.: A4

Rize: 2; toprak üzeri

P. neesii (Müll.Hal.) Dozy. - Lok.: A4 Rize: 2; toprak üzeri

P. urnigerum (Hedw.) P.Beauv. - Lok.: A4 Rize: 2, 7, 11, 21, 22, 23, 26; 1slak toprak üzeri,

Polytrichastrum alpinum (Hedw.) G.L.Sm. - Lok.: A4 Rize: 13, 15, 24, 27; toprak üzeri, 1slak toprak üzeri

Polytrichum commune Hedw. - Lok.: A4 Rize: 7, 22, 26, 29; 1slak toprak üzeri.

P. formosum Hedw. - Lok.: A4 Rize: 2, 7, 9, 13, 24, 26, 29; 1slak toprak üzeri, toprak üzeri.

P. juniperum Hedw. - Lok.: A4 Rize: 2, 11, 13, 14, $15,19,22,24,29$; toprak üzeri, 1slak toprak üzeri

P. piliferum Hedw. - Lok.: A4 Rize: 15, 28, 30, 31, 33; toprak üzeri, kaya üzeri, sslak toprak üzeri

Pottiaceae Schimp.

\#Anoectangium aestivum (Hedw.) Mitt. - Lok.: A4

Rize: 1, 6, 7, 15, 29; kaya üzeri, toprak üzeri

Barbula unguiculata Hedw. - Lok.: A4 Rize: 12; sslak toprak üzeri

Bryoerythrophyllum ferruginascens (Stirt.)

Giacom. - Lok.: A4 Rize: 25; 1slak toprak üzeri

B. recurvirostrum (Hedw.) P.C.Chen. - Lok.: A4

Rize: 1, 6, 21; kaya üzeri.

\#Chionoloma tenuirostre (Hook. \& Taylor)

M.Alonso, M.J.Cano \& J.A.Jiménez. - Lok.: A4 Rize: 22, 33; 1slak toprak üzeri.

\#Cinclidotus fontinaloides (Hedw.) P.Beauv. -

Lok.: A4 Rize: 32; 1slak toprak üzeri

Didymodon acutus (Brid.) K.Saito. - Lok.: A4 Rize:

11; 1slak toprak üzeri.

D. ferrugineus (Schimp. ex Besch.) M.O.Hill. Lok.: A4 Rize: 21; kaya üzeri.

D. luridus Hornsch. - Lok.: A4 Rize: 33; rslak toprak üzeri.

D. rigidulus Hedw. - Lok.: A4 Rize: 6; toprak üzeri.

D. vinealis (Brid.) R.H.Zander. - Lok.: A4 Rize: 3,

21; kaya üzeri

Syntrichia laevipila Brid. - Lok.: A4 Rize: 11; 1slak toprak üzeri

\#S. minor (Bizot) M.T.Gallego, J.Guerra, M.J.Cano, Ros \& Sánchez-Moya. - Lok.: A4 Rize: 25; 1slak toprak üzeri.

S. montana Nees. - Lok.: A4 Rize: 1, 5, 6, 7, 12, $14,16,18,19,21,23,29$; kaya üzeri, 1slak toprak üzeri
S. norvegica F.Weber. - Lok.: A4 Rize: 14, 15, 17,

19, 24, 29, 32, 33; kaya üzeri, toprak üzeri

\#S. papillosissima (Copp.) Loeske. - Lok.: A4 Rize:

31 ; 1slak toprak üzeri

\#S. ruraliformis (Besch.) Mans. - Lok.: A4 Rize:

15, 27; kaya üzeri, sslak toprak üzeri

S. ruralis (Hedw.) F.Weber \& D.Mohr. - Lok.: A4

Rize: 1, 2, 5, 6, 7, 8, 9, 11, 12, 14, 16, 17, 20, 24,

27, 28, 30; kaya üzeri, toprak üzeri.

\#S. virescens (De Not.) Ochyra. - Lok.: A4 Rize:

28, 30; 1slak toprak üzeri

Tortella fragilis (Drumm.) Limpr. - Lok.: A4 Rize: 6 ; toprak üzeri.

\#T. inclinata (R.Hedw.) Limpr. - Lok.: A4 Rize: 28, 30; 1slak toprak üzeri.

T. tortousa (Hedw.) Limpr. - Lok.: A4 Rize: 5, 6, 7, 11, 12, 13, 14, 15, 16, 17, 18, 20, 21, 22, 25, 27, 29, 31, 33; kaya üzeri, toprak üzeri.

\#Tortula acaulon var. pilifera (Hedw.) R.H.Zander. - Lok.: A4 Rize: 15; kaya üzeri.

T. hoppeana (Schultz) Ochyra. - Lok.: A4 Rize: 11, 13, 15, 24, 28, 30; kaya üzeri, 1slak toprak üzeri

T. marginata (Bruch \& Schimp.) Spruce. - Lok.: A4 Rize: 1, 4, 5, 8, 9, 13, 14, 15, 17, 24, 27, 29, 32, 33; kaya, toprak üzeri

\#T. mucronifolia Schwägr. - Lok.: A4 Rize: 24; toprak üzeri.

T. muralis Hedw. - Lok.: A4 Rize: 7, 29; toprak üzeri, 1slak toprak üzeri

\#T. solmsii (Schimp.) Limpr. - Lok.: A4 Rize: 21; kaya üzeri.

T. subulata Hedw. - Lok.: A4 Rize: 1, 3, 6, 7, 8, 15, 17, 21; kaya üzeri, toprak üzeri

\#T. vahliana (Schultz) Mont. - Lok.: A4 Rize: 7; toprak üzeri.

Weissia condensa (Voit) Lindb. - Lok.: A4 Rize: 25; toprak üzeri.

W. controversa Hedw. - Lok.: A4 Rize: 21, 29; kaya üzeri

Pseudoleskeaceae Schimp.

Lescuraea incurvata (Hedw.) E.Lawton. - Lok.: A4 Rize: 9, 11, 24, 29; toprak üzeri, sslak toprak üzeri. L. mutabilis (Brid.) Lindb. ex I.Hagen. - Lok.: A4 Rize: 7, 8, 11, 14, 20, 29, 33; toprak üzeri, 1slak toprak üzeri, ağaç gövdesi üzeri

L. patens Lindb. - Lok.: A4 Rize: 23; 1slak toprak üzeri

L. plicata (Schleich. ex F.Weber \& D.Mohr) Broth. - Lok.: A4 Rize: 8, 9, 11, 13, 23; 1slak toprak üzeri L. radicosa (Mitt.) Mönk. - Lok.: A4 Rize: 7, 8, 9, $11,16,22,24,29$; kaya üzeri, toprak üzeri, 1slak toprak üzeri

L. saxicola (Schimp.) Molendo. - Lok.: A4 Rize: 33; 1slak toprak üzeri.

Pseudoleskeellaceae Ignatov \& Ignatova

Pseudoleskeella nervosa (Brid.) Nyholm. - Lok.: A4 Rize: 1, 4, 7, 8, 9, 14, 17, 18, 19, 20, 21, 23, 25; 
kaya üzeri, toprak üzeri, ağaç gövdesi üzeri, sslak toprak üzeri

Pterigynandraceae Schimp.

Pterigynandrum filiforme Hedw. - Lok.: A4 Rize: $1,2,4,8,9,15,16,17,20,21,31,33$; kaya üzeri, ağaç gövdesi üzeri, ıslak toprak üzeri

Pylaisiaceae Schimp.

Calliergonella cuspidata (Hedw.) Loeske. - Lok.: A4 Rize: 14, 16, 17, 18, 19, 21, 22, 33; 1slak toprak üzeri

C. lindbergii (Mitt.) Hedenäs. - Lok.: A4 Rize: 11, 28, 30, 33; 1slak toprak üzeri

Pseudohygrohypnum eugyrium (Schimp.) Kanda. Lok.: A4 Rize: 12, 22; 1slak toprak üzeri

Pylaisia polyantha (Hedw.) Schimp. - Lok.: A4 Rize: 8; toprak üzeri.

\#Roaldia revoluta (Mitt.) P.E.A.S.Câmara \& M.Carvalho-Silva. - Lok.: A4 Rize: 7, 11, 24, 29; toprak üzeri, 1slak toprak üzeri

Pylaisiadelphaceae Goffinet \& W.R.Buck

Platygyrium repens (Brid.) Schimp. - Lok.: A4 Rize: 12; 1slak toprak üzeri.

Rhabdoweisiaceae Limpr.

\#Cynodontium fallax Limpr. - Lok.: A4 Rize: 11; sslak toprak üzeri.

\#C. jenneri (Schimp.) Stirt. - Lok.: A4 Rize: 11; toprak üzeri.

Dicranoweisia cirrata (Hedw.) Lindb. - Lok.: A4 Rize: 7, 27, 29; toprak üzeri, 1slak toprak üzeri Oncophorus virens (Hedw.) Brid. - Lok.: A4 Rize: $4,11,24$; 1slak toprak üzeri, ağaç gövdesi üzeri.

Rhytidiaceae Broth.

Rhytidium rugosum (Hedw.) Kindb. - Lok.: A4 Rize: 1, 5, 6, 7, 16, 20, 29; kaya üzeri, toprak üzeri. Saelaniaceae Ignatov \& Fedosov

\#Saelania glaucescens (Hedw.) Broth. - Lok.: A4

Rize: 7, 32; toprak üzeri

Scorpidiaceae Ignatov \& Ignatova

Sanionia uncinata (Hedw.) Loeske. - Lok.: A4 Rize: 8, 9, 11, 13, 24, 26, 29; toprak üzeri, 1slak toprak üzeri

\#Scorpidium revolvens (Sw. ex anon.) Rubers. Lok.: A4 Rize: 26; 1slak toprak üzeri.

Sphagnaceae Dumort.

\#Sphagnum capillifolium (Ehrh.) Hedw. - Lok.: A4 Rize: 24, 26, 28; su içi.

S. centrale C.E.O.Jensen. - Lok.: A4 Rize: 26; su içi.

S. contortum Schultz. - Lok.: A4 Rize: 26; su içi.

S. fallax (H.Klinggr.) H.Klinggr. - Lok.: A4 Rize: 26; su içi.

*S. fimbriatum Wilson. - Lok.: A4 Rize: 26; su içi.

S. girgensohnii Russow. - Lok.: A4 Rize: 26; su içi. S. molle Sull. - Lok.: A4 Rize: 28; su içi.

*Sphagnum papillosum Lindb. - Lok.: A4 Rize: 26; su içi.

S. platyphyllum (Lindb. ex Braithw.) Warnst. Lok.: A4 Rize: 22, 23; su içi, sslak toprak üzeri.
\#S. rubellum Wilson. - Lok.: A4 Rize: 24, 26; su içi.

S. squarrosum Crome. - Lok.: A4 Rize: 18, 26; su içi.

S. teres (Schimp.) Ångstr. - Lok.: A4 Rize: 22, 26; su içi.

S. warnstorfii Russow. - Lok.: A4 Rize: 24; su içi.

Stereodontaceae Hedenäs, Schlesak \& D.Quandt

Stereodon callichrous (Brid.) Lindb. - Lok.: A4 Rize: 16, 26, 27; kaya üzeri, 1slak toprak üzeri

\#S. hamulosus (Schimp.) Lindb. - Lok.: A4 Rize: 7 , 16 ; toprak üzeri, kaya üzeri.

Thuidiaceae Schimp.

Abietinella abietina (Hedw.) M.Fleisch. var. abietina. - Lok.: A4 Rize: 2, 4, 5, 6, 7, 20, 22; toprak üzeri, 1slak toprak üzeri, ağaç gövdesi üzeri A. abietina var. hystricosa (Mitt.) Sakurai. - Lok.: A4 Rize: 7, 8, 11, 12, 14, 16, 19, 21, 31; toprak üzeri, kaya üzeri, 1slak toprak üzeri

Thuidium assimile (Mitt.) A.Jaeger. - Lok.: A4

Rize: 1, 4, 5, 8, 19, 21; kaya üzeri, toprak üzeri.

T. delicatulum (Hedw.) Schimp. - Lok.: A4 Rize: 5, 25; toprak üzeri, 1slak toprak üzeri

T. recognitum (Hedw.) Lindb. - Lok.: A4 Rize: 17; toprak üzeri.

T. tamariscinum (Hedw.) Schimp. - Lok.: A4 Rize: 2; toprak üzeri.

\section{Tartışma ve Sonuç}

Anzer vadisi ve çevresinden 2019 yılının Haziran ve Temmuz aylarında yapılan arazi çalışmaları sonucunda toplanan yaklaşık \pm 6000 briyofit örneğinin incelenmesi sonucu, 69 familya ve 135 cinse ait toplam 338 tür, alttür ve varyete düzeyinde briyofit taksonu teşhis edilmiştir. Ciğerotlarına ait familya sayısı 21, karayosunlarında ise 48'dir. Ciğerotu taksonlarının ait olduğu cins sayısı 30 iken bu rakam karayosunları için 105 'tir. Toplam takson sayısının 286'si karayosunu, 52'si ise ciğerotu taksonlarıdır. Scapania obscura (Arnell \& C.E.O.Jensen) Schiffn., Sphagnum fimbriatum Wilson, $S$. papillosum Lindb., Dicranella staphylina H.Whitehouse ve Pohlia lescuriana (Sull.) Ochi taksonlarının Türkiye'den ikinci kez kaydı verilmiştir.

Kara ve ark. (2014) tarafından Erciyes Dağı'nda $2371 \mathrm{~m}$ rakımda toprak üzerinden toplanan ve Türkiye için ilk defa kaydı verilen Scapania obscura, bu çalışmada Türkiye için ikinci kayıt olarak verilmiştir. Sunulan bu araștırmada ayrıca, Arcahal ve Yatak yaylası'ndan olmak üzere her iki lokalitede $2500 \mathrm{~m}$ rakım üzerinde sslak toprak üzerinden toplanmıştır.

Türkiye için ikinci kayıt olarak verilen Sphagnum fimbriatum 'un ülkedeki ilk lokalite kayıt bilgileri Tonguç-Yayıntaş (2013) tarafindan Çanakkale ili 
Çan ilçesinden $650 \mathrm{~m}$ rakımda su içerisinden verilmiştir. Söz konusu tür, Anzer vadisi ve Arcahal yaylası'nda ise $2600 \mathrm{~m}$ rakımda dere kenarı islak toprak üzeri ve su içinden toplanmıştır. Örneğin ilk kaydının verildiği Çanakkale ili Çan ilçesinin vejetasyonunda Pinus nigra subsp. pallasiana (Lamb.) Holmboe., Castanea sativa Mill., Quercus petraea (Mattuschka) Liebl. ve Quercus frainetto Ten. karışık ormanı hakim iken, Arcahal yaylası'nın hakim vejetasyon tipi ise alpin çayır ve Rhododendron caucasicum Pall'dir.

Kırmacı ve ark. (2017) tarafından Türkiye için yeni kayıt olarak verilen $S$. papillosum Arhavi (Artvin)'den $1650 \mathrm{~m}$ yükseltide su içinden toplanmışken, Anzer vadisinde ise Arcahal yaylası'nda $2600 \mathrm{~m}$ de dere kenarı ıslak toprak üzeri ve su içinden toplanmıştır. Türün ülkedeki ilk kaydının verildiği Arhavi (Artvin) ilçesindeki lokalite Picea orientalis (L.) Link ve Rhododendron ponticum L. formasyonu ile kaplı iken, araştırma alanınzdaki lokalitelerden biri olan Arcahal yaylasında alpin çayır ve Rhododendron caucasicum Pall.'un yayılışı gözlemlenmiştir.

Floristik listedeki karayosunu taksonlarından biri olan Dicranella staphylina, Ursavaş ve ark. (2021) tarafından Karasu (Sakarya), Samanlı Dağları (Sakarya) ve Karacabey (Bursa)'dan Türkiye için yeni kayıt olarak verilmiştir. $\mathrm{Bu}$ çalışmada Garzavan yaylasında toprak üzerinde bulunmuştur. D. staphylina, Karasu (Sakarya), Samanlı Dağları (Sakarya) ve Karacabey (Bursa) lokalitelerinden 13 ile $769 \mathrm{~m}$. rakım aralığında toprak üzerinde toplanmışken, Garzavan yaylası'nda $2072 \mathrm{~m}$. rakımda toprak üzerinden toplanmıştır. Örneğin, yeni kayıt olarak verildiği lokalitelerde hakim vejetasyon olarak Alnus glutinosa (L.) Gaertn., A. orientalis Decne., Carpinus orientalis Mill., $C$. betulus L., Castanea sativa Mill., Corylus avellana L., Fagus orientalis Lipsky karışık orman tipi görülürken, Garzavan yaylası'ndaki lokalitede hakim vejetasyon tipi Picea orientalis (L.) Link. saf ormanıdır.

Türkiye'den ilk kez Erata ve ark. (2021) tarafindan yeni kayıt olarak verilen Pohlia lescuriana, Kümbet yaylası (Dereli, Giresun)'ndan 1630 m. rakımda dere kenarı ıslak toprak üzerinden toplanmıştır. $\mathrm{Bu}$ çalışmada Dereköy girişi, Şelale mevkii'nde 1100 m. rakımda dere kenarı 1slak toprak üzerinden kaydı verilmiştir. Kümbet yaylası vejetasyon tipi Picea orientalis (L.) Link., saf ormanı ile kaplı iken; Dereköy girişi, Şelale mevkiinin vejetasyonu ise Fagus orientalis Lipsky, Alnus glutinosa (L.) Gaertner, Castanea sativa Mill. Corylus avellena L. taksonlarından oluşmaktadır.
Türkiye briyofit florası için yeni kayıt olarak Kara ve ark. (2007) tarafindan Hatay ilinden verilen Encalypta microstoma Bals.-Criv. \& De Not.'nın ülkemizdeki diğer yayılış alanları farklı yazarlar tarafindan farklı coğrafik bölgelerden verilmiştir. Sirasiyla; Batan ve Özdemir (2011)-Mersin, Batan ve ark. (2013)-Iğdır, Kara ve ark. (2013)-Hatay (Amanos Dağları), Ezer ve ark. (2015)-Niğde, Batan ve Özdemir (2016)-Burdur ili kayıtları mevcuttur. $\mathrm{Bu}$ çalışma ile ilk kez Karadeniz bölgesinden kaydı verilmiştir.

Ayrıca, 88 briyofit ( 9 ciğerotu ve 79 karayosunu) taksonu Rize ili için ilk defa rapor edilmiştir. Rize ili için yeni olan 9 ciğerotu taksonu; Barbilophozia lycopodioides, Gymnocolea inflata, Fuscocephaloziopsis lunulifolia, Lophoziopsis excisa, Lophoziopsis longidens, Porella cordaeana, Scapania obscura, Solenostoma sphaerocarpum ve Southbya tophacea'dir. Rize ili için yeni olan karayosunları ise; Campylium bambergeri, Hygroamblystegium tenax, Platyhypnum duriusculum, P. smithii, Brachythecium campestre, B. tommasinii, Homalothecium philippeanum, Sciuro-hypnum flotowianum, S. latifolium, Anomobryum concinnatum, Bryum dichotomum, Imbribryum alpinum, Ptychostomum compactum, $P$. creberrimum, $P$. donianum, $P$. funkii, $P$. pallescens, $P$. pseudotriquetrum var. bimum, $P$. rubens, $P$. weigelii, Warnstorfia fluitans, Dicranum brevifolium, D. elongatum, D. leioneuron, $D$. spadiceum, Dicranella staphylina, D. varia, Encalypta microstoma, Fontinalis squamosa, Grimmia anodon, G. anomala, G. caespiticia, $G$. donniana, G. lisae, G. orbicularis, G. unicolor, Racomitrium sudeticum, Schistidium agassizii, S. brunnescens subsp. brunnescens, S. brunnescens subsp. griseum, S. confusum, S. dupretii, $S$. elegantulum, S. platyphyllum, $S$. rivulare, Rhytidiadelphus subpinnatus, Hypnum resupinatum, Mnium spinosum, $M$. spinulosum, $M$. thomsonii, Pohlia lescuriana, Rhizomnium magnifolium, R. pseudopunctatum, Orthotrichum pulchellum, O. tenellum, Ulota crispula, Pseudotaxiphyllum elegans, Anoectangium aestivum, Chionoloma tenuirostre, Cinclidotus fontinaloides, Syntrichia minor, S. papillosissima, S. ruraliformis, S. virescens, Tortella inclinata, Tortula acaulon var. pilifera, T. mucronifolia, $T$. solmsii, T. vahliana, Roaldia revoluta, Cynodontium fallax, C. jenneri, Saelania glaucescens, Scorpidium revolvens, Sphagnum capillifolium, $S$. fimbriatum, $S$. papillosum, $S$. rubellum ve Stereodon hamulosus dur.

Türkiye'de en fazla briyofloristik çalışmaların yapıldığı illerden biri Rize ili olmasına karşın, yapılan bu çalışma sonucunda Rize ili için yeni olan 
88 taksonun eklenmesi ile Rize ilinden tespit edilmiş olan briyofit taksonu sayısı 424'e çıkmıştır. Bu da detaylı çalışmalar sonucunda il, bölge ve ülke bazında çok sayıda yeni taksonların eklenebileceğini göstermektedir.

Çalışma sonucunda tespit edilen ciğerotu familyaları içerisinde en fazla cins içeren familyalar; Lophoziaceae (3) ve Scapaniaceae (3)'dir. İçerdikleri takson sayısı bakımından en zengin ciğerotu cinsleri ise Scapania (7), Barbilophozia (3), Lophocolea (3), Metzgeria (3) ve Solenostoma (3)'dır. Takson çeşitliliği açısından en zengin familyalar incelendiğinde, ciğerotları içerisinde 10 taksona sahip Scapaniaceae familyasını 5 taksonla Lophocoleaceae familyasının izlediği görülmektedir. Karayosunu familyaları içerisinde en fazla cins içeren familyalar; Brachytheciaceae (10), Pottiaceae (10) ve Amblystegiaceae (8)'dir. İçerdikleri takson sayısı bakımından en zengin cinsler ise; Grimmia (17), Ptychostomum (16), Schistidium (15) ve Sphagnum (13)'dur. Takson çeşitliliği açısından karayosunlarının ait oldukları familyalara bakıldığında; Grimmiaceae'nin sahip olduğu 39 taksonla floristik listede ilk sırada yer aldığ 1 tespit edilmiştir. $\mathrm{Bu}$ familyayı Pottiaceae (32), Brachytheciaceae (26), Bryaceae (22) ve Mniaceae (21) gibi familyalar takip etmektedir.

Çalışma alanında çok yaygın olarak tespit edilen taksonlar; Conocephalum conicum, Frullania tamarisci, Marchantia polymorpha, Pellia epiphylla, Plagiochila asplenioides, Bartramia halleriana, Philonotis fontana, Brachythecium rivulare, B. rutabulum, Eurhynchium angustirete, Homalothecium philippeanum, Sciuro-hypnum populeum, Ptychostomum moravicum, $P$. pseudotriquetrum var. pseudotriquetrum, Dicranum scoparium, Entodon concinnus, Grimmia hartmanii, Racomitrium canescens, Schistidium apocarpum, S. papillosum, Hylocomiadelphus triquetrus, Hylocomium splendens, Hypnum cupressiforme var. cupressiforme, Isothecium alopecuroides, Leucodon sciuroides, Mnium spinosum, Rhizomnium punctatum, Alleniella complanata, Lewinskya rupestris, Ulota crispula, Pogonatum urnigerum, Polytrichum formosum, Syntrichia montana, S. ruralis, Tortella tortousa, Tortula marginata, T. subulata, Lescuraea mutabilis, Pseudoleskeella nervosa, Calliergonella cuspidata, Sanionia uncinata, Abietinella abietina var. hystricosa ve Thuidium assimile'dir.

Çalışma alanında çok fazla yaygın olmayan taksonlar ise; Calypogeia fissa, Mesoptychia bantriensis, Lejeunea cavifolia, Telaranea europaea, Lophocolea bidentata, Lophoziopsis excisa, Scapania nemorea, Solenostoma gracillimum, Hygrohypnum luridum, Platyhypnum molle, Amphidium mougeotii, Antitrichia curtipendula, Brachythecium campestre, $B$. tommasinii, Rhynchostegium confertum, Sciurohypnum latifolium, Anomobryum concinnatum, Ptychostomum weigelii, Sarmentypnum exannulatum, Straminergon stramineum, Warnstorfia fluitans, Dicranum brevifolium, D. leioneuron, Encalypta microstoma, Fissidens bryoides, Fontinalis squamosa, Grimmia anodon, G. unicolor, Schistidium agassizii, Mnium hornum, Pohlia lescuriana, P. ludwigii, Lewinskya affinis, Orthotrichum alpestre, O. tenellum, Pseudotaxiphyllum elegans, Pogonatum aloides, Barbula unguiculata, Cinclidotus fontinaloides, Didymodon acutus, Syntrichia minor, $S$. papillosissima, Tortula acaulon var. pilifera, $T$. vahliana, Weissia condensa, Lescuraea patens, Cynodontium fallax ve Scorpidium revolvens'dir.

\section{Teşekkürler}

FBA-2019-1005 proje kod numaralı bu çalışmaya desteklerinden dolayı Recep Tayyip Erdoğan Üniversitesi Bilimsel Araştırma Projeleri Koordinatörlüğüne teşekkürlerimizi sunarız. Ayrıca, arazi çalışmalarının yapılması ve briyofit örneklerinin toplanması için gerekli izni uygun gören T.C. Tarım ve Orman Bakanlığı Doğa Koruma ve Milli Parklar Genel Müdürlüğü'ne teşekkür ederiz.

\section{Kaynaklar}

Abay G. Batan N. Özdemir T. 2016. Bryophyte Checklist of Rize, North-East Turkey. Arctoa. 25: 386-392.

Abay G. 2017. A preliminary list of subalpine and alpine bryophytes of Rize, North-East Turkey. Anatolian Bryology. 3:2, 75-80.

Abay G. 2018. The Bryophyte Flora of Recep Tayyip Erdoğan University, Zihni Derin Campus (Rize-Turkey). Anatolian Bryology. 4:2, 72-78.

Akman Y. 1999. İklim ve Biyoiklim (Biyoiklim Metodları ve Türkiye İklimleri). Kariyer Matbaacılık. Ankara.

Anşin A. 1981. Doğu Karadeniz Bölgesi Sahil ve İç Kesimlerinde Yayılan Ana Vejetasyon Tipleri. KTÜ Orm. Fak. Derg. 4: 15-64.

Batan N. Özdemir T. 2011. Mersin (C12), Trabzon ve Gümüşhane (A4)' den bazı karayosunu (musci) kayıtları. SDÜ Orman Fakültesi Dergisi. 12: 104-109.

Batan N. Özdemir T. 2016. The Bryophyte Flora of Burdur Province (Turkey). Arctoa. 25: 160170. 
Batan N. Özdemir T. Alataş M. 2013. Bryophyte flora of the Iğdır province (Turkey). Phytologia Balcanica. 9:2, $179-191$.

Batan N. Atamov V. Ekşi S. Erata H. 2018. Contrubition to the bryophyte flora of the İkizdere district (Rize, Turkey). Phytologia Balcanica. 24:1, 9-15.

Brugués M. Cros R.M. Guerra J. 2007. Flora Briofitica Ġbérica Volume I, Uniersidad de Murcia, ISBN: 978-84-611-8462-0. Sociedad Espanola de Briyologia Murcia. Murcia.

Brugués M. Guerra J. 2015. Flora Briofitica Ibérica Volume 2, Uniersidad de Murcia, ISBN: 84608-2198-4, Sociedad Espanola de Briyologia Murcia, Murcia.

Casas C. Brugués M. Cros M.R. Sérgio C. Infante M. 2009. Handbook of Liverworts and Hornworts of The İberian Peninsula and The Balearic Islands, ISBN: 978-84-92583-55-3, 177 p, Institut D'estudis Catalans, Spain.

Cortini Pedrotti C. 2001. Flora dei muschi d'ee Italia, Sphagnopsida, Andreaopsida, Bryopsida (I parte). ISBN: 88-7287-250-2, Antonio Delfino Editore Medicina-Scienze.

Cortini Pedrotti C. 2006. Flora dei muschi dee Italia, Bryopsida (II parte), ISBN: 88-7287-370-3, Antonio Delfino Editore Medicina-Scienze, Roma.

Crum A.H. Anderson E.L. 1981. Mosses of Eastern North America. Columbia University Press. Newyork.

Erata H. Öztürk-Özen Ö. Batan N. Alataş M. 2021. Pohlia Hedw. and Oleolophozia L. Söderstr., De Roo \& Hedd. Species New to Turkey and South-West Asia. Cryptogamie Bryologie. 42:1, 1-10.

Ezer T. Kara R. Seyli T. Ertek A. 2015. The Bryophyte Flora of Aladağlar National Park (Turkey). Folia Cryptogamie Estonica. 52: 7-20

Frey W. Frahm J.P. Fischer E. Lobin W. 2006. The liverworts, mosses and ferns of Europe. Essex. Harley Books.

Guerra J. Cano M.J. Cros R.M. 2006. Flora Briofitica Ibérica Volume 3, Uniersidad de Murcia, ISBN: 84-609-9097-4. Sociedad Espanola de Briyologia Murcia. Murcia.

Hedenas L. 1992. Taxonomic studies on pleurocarpous mosses, with specialreference to the Calliergon-ScorpidiumDrepanocladus complex in northern Europe. Stockholm.

Hodgetts N.G. Söderström L. Blockeel T.L. Caspari S. Ignatov M.S. Konstantinova N.A. Lockhart N. Papp B. Schröck C. SimSim M. ve ark. 2020. An annotated checklist of bryophytes of Europe, Macaronesia and Cyprus. Journal of Bryology. 42:1, 1-116.

Kara R. Ezer T. Düzenli A. Erdağ A. 2007. New national and regional bryophyte records, 15. Journal of Bryology. 29: 139-142.

Kara R. Ezer T. Düzenli A. 2013. The Bryophyte Flora of Northern Amanos (Nur) Mountain (Hatay-Turkey). Evansia. 30:1, 1-14.

Kara R. Ezer T. Can Gözcü M. Bozdoğan Ş.G. 2014. Bryophyte flora of Erciyes Mountain in Turkey, with 6 bryophyte records from the country. Turkish Journal of Botany. 38: 763781.

Kirmaci M. Semiz A. Şen A. 2017. Türkiye Sphagnum L. (Sphagnaceae) Cinsinin Revizyonu. TÜBITTAK projesi. Andan Menderes Üniversitesi. Aydın.

Kürschner H. Frey W. 2020. Liverworts, mosses and hornworts of Southwest Asia (Marchantiophyta, Anthoceratophyta, Bryophyta). Nova Hedwigia. 149: 1-267.

Nyholm E. 1986. Illustrated Flora of Nordic Mosses, Fasc. 1. Fissidentaceae Seligeriaceae, 1-72, The Nordic Bryological Society, Lund.

Nyholm E. 1989. Illustrated Flora of Nordic Mosses, Fasc. 2. Pottiaceae - SplachnaceaeSchistostegaceae, 75-141, The Nordic Bryological Society, Lund.

Nyholm E. 1993. Illustrated Flora of Nordic Mosses, Fasc. 3. Bryaceae-Rhodobryaceae Mniaceae -Cinclidiaceae-Plagiomniaceae. 145-244, The Nordic Bryological Society, Lund.

Nyholm E. 1998. Illustrated Flora of Nordic Mosses, Fasc. 4. AulacomniaceaeMeesiaceae-Catocopiaceae-BartramiaceaeTimmiaceae-Encalyptaceae-Grimmiaceae Ptychomitriaceae-HedwigiaceaeOrthotrichaceae. 145-244, The Nordic Bryological Society, Lund.

Paton J. 1999. The Liverworts Flora of the British Isles, ISBN: 0-946589-60-7, 626 pp, Harley Books, England.

Smith A.J.E. 1996. The Liverworts of Britain and Ireland, ISBN: 0-521-42473-9, 384 pp Cambridge University Press, Cambridge.

Smith A.J.E. 2004. The Moss Flora of Britain and Ireland. Second Edition, ISBN: 0-521816408, 1012 pp, Cambridge University Press, Cambridge.

Tonguc Yayintas Ö. 2013. New Moss Records From Western Part of Turkey. Bangladesh Journal of Botany. 42:2, 371-375.

Ursavaş S. Keçeli T. Uyar G. Ören M. 2021. Dicranella staphylina (Dicranaceae), a new moss record from Turkey and South West Asia. Plant Biosystems. 155:3, 483-486. 